\title{
Dynamic Pricing of Peak Production
}

\author{
Heikki Peura, Derek W. Bunn \\ London Business School, Regent's Park, London NW1 4SA, United Kingdom, hpeura@london.edu, dbunn@london.edu
}

Peak producers of non-storable products, such as electricity, provide crucial flexible operating capacity to respond to infrequent and transient high demand periods. Faced with these uncertain revenue-earning opportunities, despite often having significant price-setting power, they need to profit from a limited number of pricing decisions in order to meet financial targets for viability. We study the repeated interaction between peak producers with a model that captures both the uncertainty in their short-term revenues and their market power. We investigate the conditions under which peak producers can implicitly co-ordinate to achieve high prices, under varying demand conditions. We analyze how financial objectives in the form of annual performance targets dynamically impact peakers' pricing decisions, and the conditions under which setting such targets may benefit or hurt the owners of the firm. We further show how portfolio integration with lower marginal cost technologies can be an important factor in peak price setting, beyond the usual considerations of direct price externalities, capacity manipulation or risk. These insights are useful not only in understanding how purely energy-based revenues can sustain the financial viability of peakers, and the dynamic emergence of price spikes, but also in providing the underlying process for pricing derivative contracts that policy makers may encourage or offer for resource adequacy.

Key words: noncooperative games; energy; electric industries

\section{Introduction}

Many supply-chain, product or service pricing decisions are characterized by capital-intensive facilities having random, infrequent and transient revenue-earning opportunities, without any inventories for production smoothing. Examples of such situations range from the repetitive competitive bidding that many specialized engineering and construction companies have to undertake as their business model, or event promoters organizing only a few high-profile events per year, to managing an electricity generating plant that may only operate at times of scarcity. The key features of such problems often include (i) supplier market power in the repeated, yet infrequent, interaction of a small number of peak producers and (ii) significant revenue uncertainty and the need to profit from a limited number of earning opportunities in order to meet financial targets for viability. In this paper, we study how these counterbalancing forces affect the pricing decisions of suppliers.

An important example of this class of problems is in the electricity industry, with crucial implications (such as large-scale blackouts) to society if it is not solved efficiently. Electricity generation is characterized by inelastic demand, a lack of economic storage options and a concentrated industry 
structure, creating opportunities for suppliers to exercise market power under scarcity. In these situations, prices are sometimes set by peaking plants, which are typically low capital cost, high marginal cost generators with operational flexibility, whilst normally acting as idle reserve capacity. Given the infrequency of their opportunities to generate, they may need to recover their fixed costs for an entire year during a few hours of operation, but may indeed be able to achieve this by creating high price spikes. Whilst owners of peaking facilities may wish to use derivatives, if they are available, to manage their revenue risks, and policy makers may wish to contract with peakers for resource security, the pricing of such instruments still requires a fundamental analysis of how peak producers can otherwise behave in the underlying energy spot market. Moreover, with the increase in renewable generation pushing many traditional combustion plants into infrequent operation (Traber and Kemfert 2011; Wiesmann and Clark 2012), it is essential to understand the processes underlying the emergence of spikes through the price setting of peaking plants.

We motivate these pricing decisions through an example of the operation of peaking plants in the British electricity market. In July 2006, high temperatures combined with plant outages forced the system to run at almost full production capacity at peak times, with the System Operator issuing several warnings of demand control measures (Figure 1). The generation units at the top of the marginal cost supply stack were, at that time, three oil-powered plants owned by two companies with large generation portfolios, RWE and Eon. Normally mostly idle (with average capacity utilisation about $3 \%$ in 2006), these plants were however needed to serve demand relatively often during that month. (Although this example focuses on oil-fired plants, it would also apply to other types of peaking units, for example the least efficient gas-fired plants in a market.) In Figures 1(a) and 1(c), the half-hourly utilisations and offer prices of these plants in the real-time balancing market $^{1}$ are displayed for 18 July, 2006, one of the first days of significant scarcity. Grain (Eon) had sold much of its production forward for the peak hours and was consequently offering only a small volume during the scarcity period (at an opportunistically high price of $£ 2000 / \mathrm{MWh}$, but still less than the price cap of $£ 9999 / \mathrm{MWh}$ ). Meanwhile, the RWE-owned Fawley and Littlebrook plants were operating close to capacity at prices around $£ 500$, about $10 \mathrm{x}$ marginal cost but evidently not fully responding to the price-setting opportunity.

Eight days later, on another day of almost identical scarcity, both companies were more reactive to the system situation. Whilst Littlebrook's production had been sold forward for an unknown price that day, in Figures 1(b) and 1(d), both Grain and Fawley were able to achieve significantly higher prices than eight days earlier, with prices fairly closely in line with one another. In total, Grain was paid an exceptional $£ 2,350,000$ and Fawley $£ 1,470,000$ in one day. It is difficult to infer from the data whether the variation in pricing in the two days was due to the accuracy of demand forecasts, some kind of learning by the producers, or simply the randomness of the price-influencing 
Figure 1 Half-hourly oil plant offer prices and capacity utilisation in the UK balancing mechanism for 18 July and 26 July, 2006.

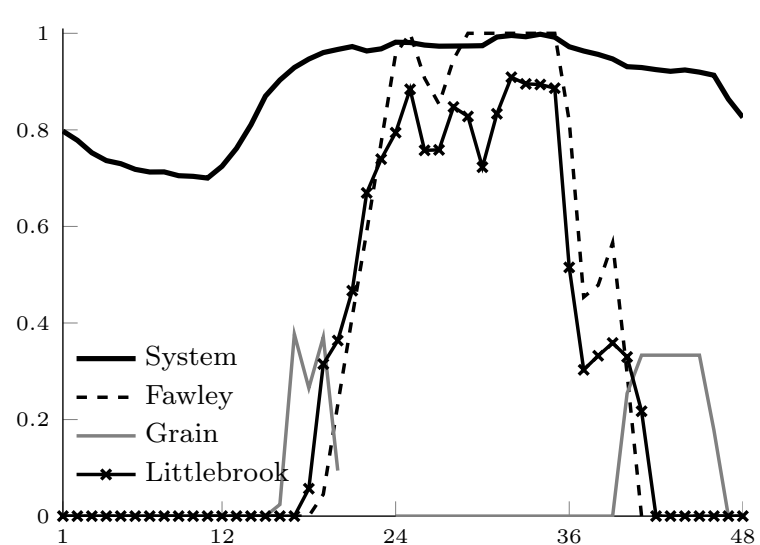

(a) 18 July 2006, capacity utilisation

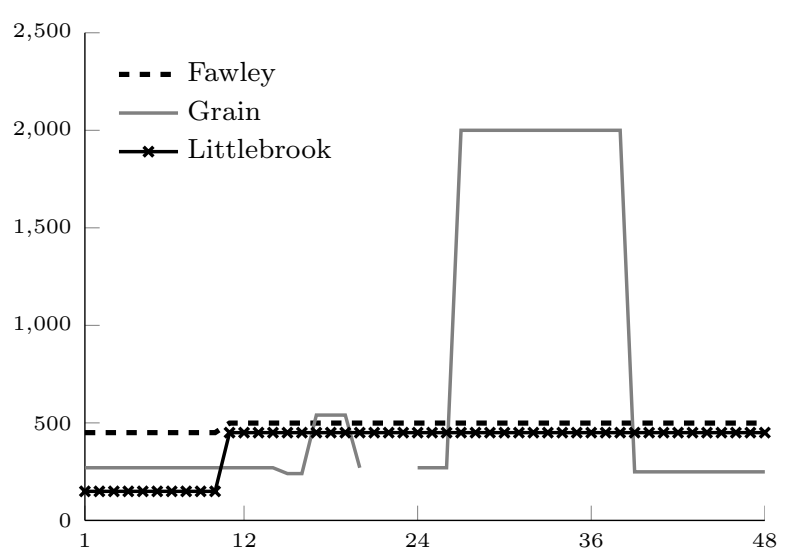

(c) 18 July 2006, offer prices (£)

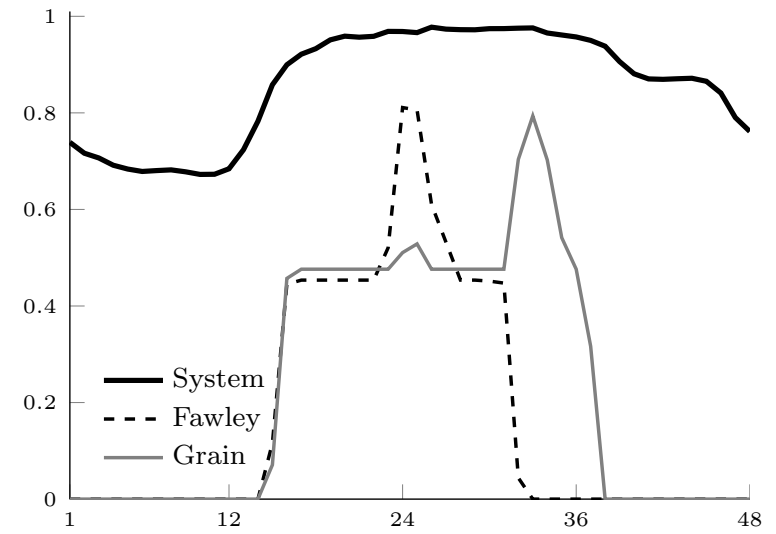

(b) 26 July 2006, capacity utilisation

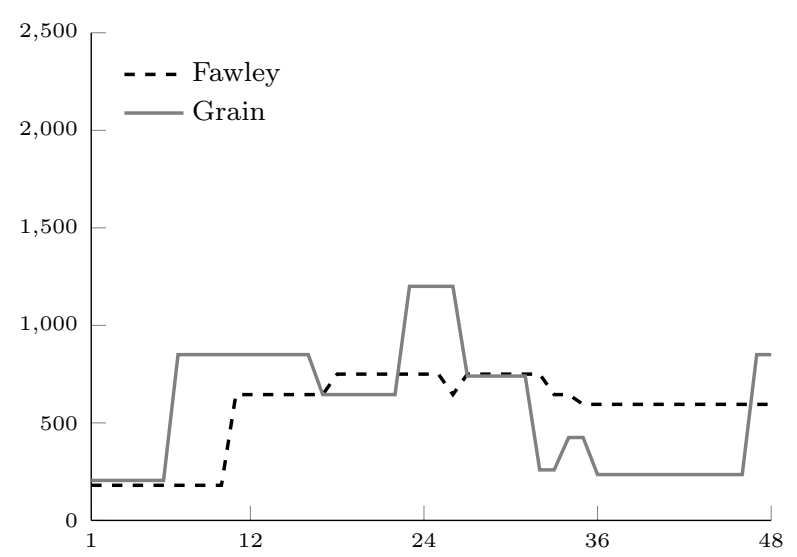

(d) 26 July 2006, offer prices (£)

processes. We can, however, observe some common features: (i) very high offer prices on both days, implying both market power and high variability in revenues; (ii) an ambiguous relationship between available capacity and pricing; and (iii) overall similar (relatively constant during the peak period) price levels between plants, suggesting some level of coordination in price setting. In sum, these and similar observations suggest fairly complex dynamics in pricing and the emergence of spikes, which may in general also depend on the long periods of idleness that peaking units often face.

This paper therefore develops a theoretical analysis of potential dynamic coordination of extreme offers over infrequent repetitions. Our model captures several features particular to the peaker setting. Firstly, peaking plants typically operate as a small oligopoly over the residual demand not fulfilled by baseload capacity. With recurring interactions among a small number of competitors, we might thus expect implicitly collusive behaviour, evidence of which in different electricity 
markets has been provided by Macatangay (2002), Guan et al. (2002), Fabra and Toro (2005) and Sweeting (2007). Secondly, due to the transient nature of peaking demand, there is significant uncertainty over future revenue-earning opportunities. Consequently, thirdly, peak producers need to meet financial viability targets during these limited opportunities, with potential penalties or even threats of closure for underperformance. We incorporate this objective through the application of periodic revenue targets, which effectively create an intertemporal externality by linking plant revenues over time. Finally, peaking plants often belong to diversified production portfolios, creating a further potential portfolio externality.

We make contributions on two fronts. Firstly, this is, to our knowledge, the first paper to identify the above key factors in modelling competition between peak producers. We show the impact of these factors on pricing, and provide insights into the occurrence of price spikes. In particular, the intertemporal externality produces distinct non-monotonic dynamic patterns in collusive and non-collusive pricing, even with stationary demand. We also demonstrate how market structure influences prices through the portfolio externality. Furthermore, collusive prices depend non-monotonically on both peak demand and capacity, which may help explain empirical patterns in price spikes; we present some indicative evidence of pricing behaviour consistent with our predictions from the British market.

Secondly, methodologically, we extend the literature on repeated price competition to consider the effects of two aspects of risk management: financial targets and technological portfolios. Targets significantly change the nature of the price game by making pricing incentives state and time dependent, and may either soften or harden price competition. Moreover, the performance-target model can be interpreted as a dynamic game of accumulating stock variables (here profits), and may be useful in various other (e.g., inventory-related) contexts. Production portfolios, on the other hand, are prevalent in the electricity context in particular, but their impact on repeated competition is not well understood.

Our analysis is divided into three parts. In Section 2, we formulate a model of peaker interaction as a repeated price game with uncertainty over both the occurrence and magnitude of future peak demand events. We derive the most-collusive equilibrium of this game as well as conditions for its existence, and show the impact of market conditions, the frequency of peaker interaction, the distribution of demand and available peaking capacity on collusive pricing.

In Section 3, we study the impact of revenue targets on pricing through dynamic incentives - the intertemporal effect. With highly uncertain revenues over a financial year, managers of peaking plants may be incentivized by performance targets, either to reach a certain profit level (for bonuses), or to at least cover fixed costs. In practice, failure to achieve such targets regularly leads to mothballing the underperforming power plants, or even downgrades from the ratings 
agencies. Underperformance of specific assets may further lead to the necessity of asset impairment provisions being reported in annual or semi-annual financial statements. These provisions affect equity values and companies are reluctant to announce them to their investors (Ernst \& Young 2013). This aversion to underperformance is particularly relevant for peaking units, as strategic decisions such as mothballing cannot readily be based on ex-ante information (such as forward curves) but must necessarily depend on ex-post information (such as actual performance compared to a target). Incorporating financial performance targets in our model, we distinguish between dynamic incentives resulting from penalties (e.g. loss of bonuses) for missing targets and the threat of mothballing or retirement. We show how revenue targets affect both non-collusive and collusive pricing in the dynamic game, and discuss their use as managerial incentives based on these results.

Finally, in Section 4, to study the portfolio effect, we allow each generating company to own both base load (low marginal cost) and peak capacity. In the discriminatory auction format typical for peaker settings, where each accepted plant is paid its own offer price, there is no clear price externality on lower marginal cost plants (unlike a uniform-price auction, in which each plant is paid the highest accepted offer, see e.g., Wolfram 1998). Therefore, it is plausible that specialization in different technologies would allow generation companies to reach higher profits. However, we find that portfolio generators achieve higher collusive prices, as they have similar incentives to coordinate pricing. Section 5 concludes, with derivations of results included in the online Electronic Companion.

\subsection{Related Literature}

While there is a large body of research on strategic interaction of producers on electricity markets, ranging from multi-unit auction (von der Fehr and Harbord 1993, Banal-Estañol and RupérezMicola 2009) to quantity competition (Jing-Yuan and Smeers 1999, Hobbs 2001, Bushnell 2003, Garcia et al. 2005, Hobbs and Pang 2007) and supply function (Green and Newbery 1992, Anderson and Philpott 2002, Anderson and Cau 2009) models, competition between peaking plants in particular has received little attention.

Although focused on the more operational setting of peak demand fluctuating over time, our work is related to economic models of repeated competition over the business cycle, which include deterministic cycles (Haltiwanger and Harrington 1991, Fabra 2006) and random demand (Rotemberg and Saloner 1986). Our context of uncertain and difficult-to-forecast peak demand in particular resembles the latter. Rotemberg and Saloner, studying collusive pricing with fluctuating elastic demand, find that "price wars", or lower than monopolistic prices, occur during booms (high demand periods). Staiger and Wolak (1992) show that this impact can be reversed with capacity constraints, and endogenize capacity choice in the collusive game. We build on these results, 
showing the impact of the infrequency of peaker interaction and the distribution of inelastic peak demand on collusion, and extend our model to include financial targets and technological portfolios. Recently, the literature on collusive pricing has been extended to cover simultaneous choice of prices and capacities (e.g., Dechenaux and Kovenock 2007, 2011). By contrast, we treat capacity constraints as exogenously given, noting that in the scarcity of the peaking context, all generators are under intense regulatory scrutiny to offer all available capacity, and in conditions of system stress, even forward contracts for export may be curtailed by the system operator (Ofgem 2013).

The impact of financial pressure on a firm in oligopolistic competition is commonly articulated in terms of debt coverage (e.g., Brander and Lewis 1986, Bolton and Scharfstein 1990, Maksimovic 1988). The effect of debt can in general be twofold: the implied threat of bankruptcy can reduce the level of competition, but debt can also drive more aggressive behaviour among firms through the effect of limited liability. For mature peaking plants, which may be fully amortized, debt as a driving force in pricing decisions appears less plausible, while financial constraints may instead take the form of performance targets. This approach has been explored in recent revenue management research, concerned with dynamic pricing of a fixed inventory over a finite time horizon, with important applications in for example airline ticket sales and retailing (see e.g., Talluri and Van Ryzin 2004). Levin et al. (2008) and Besbes and Maglaras (2012) consider dynamic pricing for a monopolistic firm facing infrequent opportunities, incorporating revenue targets via risk constraints. While both inventory constraints and the lack of competition make these models different from our peak pricing problem, they are related to ours in the context of pricing under performance constraints.

The effect of asymmetric capacity constraints on competition has been studied by several authors (e.g., Compte et al. 2002, Anderson and Cau 2011). In this vein, Knittel and Lepore (2010) show that the marginal cost of capacity may significantly impact collusion over demand cycles. Meanwhile, diversification to different markets has been found to facilitate collusion to some extent (e.g., Bernheim and Whinston 1990). However, the impact of diversification of production portfolios, with asymmetry in both capacity and marginal cost on pricing has received relatively little attention, with the exception of Banal-Estañol and Rupérez-Micola (2009), who use computational learning methods to study how generation portfolios may impact pricing in the one-shot uniformprice auction format, with a clear price externality from the highest offering plant. We, by contrast, consider the discriminatory format in a repeated setting, allowing for collusive outcomes.

\section{A Model for Peaker Pricing}

We model the strategic interaction of peak producers as an infinitely repeated discriminatory auction with uncertainty over future demand. We abstract from modelling the market in full by 
assuming that peakers serve residual demand unfulfilled by lower marginal cost plants. This allows us to consider the peakers as playing an essentially separate game from the rest of the market (we return to this assumption in Section 4). We focus on the discriminatory auction, where each winning offer is paid its offer price, as it is a format commonly employed (e.g., in Britain) in balancing markets where peakers tend to operate.

The peak producers interact repeatedly in the following stage game. In a symmetric duopoly, with marginal $\operatorname{costs} c^{i}=0$ and maximal production capacities $k^{i}=k, i \in\{1,2\}$, the producers first observe a demand realization $D \in[\underline{D}, \bar{D}]$ drawn from the distribution $F(D)$. We let $\underline{D}=0$ for convenience, and assume $\bar{D} \leq 2 k(\bar{D} \leq k$ corresponds to an uncapacitated game, where each firm can always serve the entire demand). The producers then simultaneously submit offer prices $p^{i}$ for their entire capacities to the market which is cleared by an independent system operator (SO). (As the $\mathrm{SO}$ is the counterparty for this continuous buying and selling of balancing power to meet its short-term demand forecasts, there is no demand elasticity in achieving this requirement, although it is possible in some markets that peakers may compete with demand-side offers.) Prices are constrained by a price cap $\bar{P}$, which can be interpreted as either imposed by the market regulator or as the price at which new entry would occur.

In this one-shot price game, producer $i$ maximizes its profit:

$$
\begin{aligned}
& \max _{p^{i}} \pi^{i}(D, p)=p^{i} q^{i}(D, p), \\
& q^{i}(D, p)= \begin{cases}\min \{D, k\} & \text { if } p^{i}<p^{j} \\
D / 2 & \text { if } p^{i}=p^{j} \\
\min \{\max \{0, D-k\}, k\} & \text { if } p^{i}>p^{j}\end{cases}
\end{aligned}
$$

given the offer price $p^{j}$ of the other player. Here, $q^{i}(D, p)$ is the demand served by producer $i$ given the demand and $p=\left(p^{i}, p^{j}\right)$. The equilibrium strategies in the one-shot game are well known (e.g., Fabra et al. 2006): for $D \leq k$, the (Bertrand) equilibrium price equals marginal cost: For $D>k$, there is no pure strategy equilibrium, but a unique mixed equilibrium, with expected profits increasing from competitive profits to shared monopoly profits as $D$ is increased from $k$ to $2 k$.

LEMma 1. The equilibrium of the one-shot game is as follows:

1. If $D \leq k, p^{i}=0, i=1,2$, and expected profits $\pi_{o}(D)=0$.

2. If $k<D \leq 2 k$, the equilibrium is in mixed strategies with distributions

$$
H_{o}^{i}\left(p^{i}, D\right)=H_{o}\left(p^{i}, D\right)=\frac{p^{i} k-\bar{P}(D-k)}{p^{i}(2 k-D)},
$$

with $p^{i} \in[\bar{P}(D-k) / k, \bar{P}]$, and expected profits $\pi_{o}(D)=\bar{P}(D-k)$. 
We assume that peaking demand is independently and identically distributed across periods: in each period, the plants face residual demand from the rest of the market with probability $\alpha$, which is then distributed according to $F(D)$. In the infinitely repeated game, while observing the current demand realization before making their offers, the producers only know the distribution $F(D)$ from which future realizations will be drawn. Whilst normal demand levels are well-known to be autocorrelated, the peak episodes that give rise to price spikes are short-lived, often within a day, as evident in the stochastic jump and regime-switching models which have been estimated on the time series (e.g., Mount et al. 2006).

\subsection{Equilibrium Analysis}

In the repeated game, peakers may earn collusive markups as the short-term temptation to undercut a high price is balanced against the threat of a punishment through lower future prices. Following the standard approach for repeated games, we focus on such trigger strategies, where a deviation from (implicitly) collusive pricing will cause a Nash-reversion to the one-shot equilibrium. We shall in particular derive the most-collusive equilibrium of the repeated game, that is, the highest prices that can be supported in a symmetric subgame-perfect equilibrium. Given a demand realization $D$, the plants will collude if expected punishment $M$ exceeds deviation gain $W$ :

$$
W(D)=\pi_{d}(D)-\pi_{c}(D) \leq M=\sum_{\tau=1}^{\infty} \delta^{\tau} \mathbb{E}\left[\pi_{c}-\pi_{o}\right]
$$

Here $\left(\pi_{c}, \pi_{d}, \pi_{o}\right)$ denote collusive, deviation and one-shot profits, respectively, and $\delta \in(0,1)$ is the common discount factor. Note that $M$ is independent of the current demand realization, as it is the expectation of future profits.

Let us examine deviations from highest possible markups $(p=\bar{P})$. With low demand $(D \leq k)$, a firm can capture the entire demand by undercutting; with high demand $(D>k)$, it can only supply demand up to its capacity $k$. The deviation gain is hence

$$
W(D)= \begin{cases}D \bar{P} / 2 & \text { if } D \leq k \\ (k-D / 2) \bar{P} & \text { if } D>k\end{cases}
$$

This is maximized at $k$, and deviation gains are non-monotonic: deviation is most attractive when the current period demand is neither high or low. Hence, for demand realizations between $\underline{D}^{*}$ and $\bar{D}^{*}$, collusive prices are lower than $\bar{P}$ so that deviation gains remain below $M$. The highest achievable prices and the corresponding collusive profits are given in the following Lemma.

Lemma 2. The most-collusive equilibrium in the infinitely repeated game is as follows.

If $M \leq k \bar{P} / 2$, there exists a $\underline{D}^{*}$ such that for $D \in\left[0, \underline{D}^{*}\right]$, the punishment exceeds deviation profits at price $\bar{P}$, and for $D \in\left(\underline{D}^{*}, k\right]$, deviation profits exceed the punishment. The collusive price is $\bar{P}$ for $D \in\left[0, \underline{D}^{*}\right]$ and decreasing for $D \in\left(\underline{D}^{*}, k\right]$. 
If, further, $\underline{D}^{*} \geq 2 k-\bar{D}$, there exists a $\bar{D}^{*}$ such that for $D \in\left(k, \bar{D}^{*}\right]$, deviation profits exceed the punishment at price $\bar{P}$, and for $D \in\left(\bar{D}^{*}, \bar{D}\right]$, the punishment exceeds deviation profits. In this case, $\underline{D}^{*}+\bar{D}^{*}=2 k$. The collusive price is $\bar{P}$ for $D \in\left(\bar{D}^{*}, \bar{D}\right]$ and increasing for $D>k$ otherwise.

If $\underline{D}^{*} \leq(6-4 \sqrt{2}) k$ and $\bar{D} \geq 3 k / 2-\underline{D}^{*} / 4$, there exists a range $[\hat{D}, \check{D}]$ where the one-shot mixed strategy equilibrium is played instead of collusive prices.

The equilibrium prices and profits are summarized in the table below, with $P(D)=\underline{D^{*}} \bar{P} /(2 k-D)$.

Table 1 Collusive prices and profits.

\begin{tabular}{lcc}
\hline & $p_{c}$ & $\pi_{c}(D)$ \\
\hline$D \in\left[0, \underline{D}^{*}\right]$ & $\bar{P}$ & $D \bar{P} / 2$ \\
$D \in\left(\underline{D}^{*}, k\right]$ & $\underline{D}^{*} \bar{P} / D$ & $\underline{D}^{*} \bar{P} / 2$ \\
$D \in(k, \hat{D}]$ & $P(D)$ & $D P(D) / 2$ \\
$D \in(\hat{D}, \check{D}]$ & $p^{i} \sim H_{o}\left(p^{i}, D\right)$ & $\bar{P}(D-k)$ \\
$D \in\left(\check{D}^{*} \bar{D}^{*}\right]$ & $P(D)$ & $D P(D) / 2$ \\
$D \in\left(\bar{D}^{*}, \bar{D}\right]$ & $\bar{P}$ & $D \bar{P} / 2$ \\
\hline
\end{tabular}

For $D \leq \underline{D}^{*}$ and $D \geq \bar{D}^{*}$, the producers offer the monopoly price $\bar{P}$. For $\underline{D}^{*}<D<\bar{D}^{*}$, they can at most achieve profits that imply deviation gain $M$, so prices are adjusted down accordingly. The collusive price is thus (weakly) decreasing in $D$ when $D \leq k$, and (weakly) increasing in $D$ when $D>k$. However, for $D>k$, the collusive prices may not always be sustained since the firms may prefer deviating up to $\bar{P}$ to gain higher profits. The firms then play the one-shot mixed equilibrium strategies even in the collusive equilibrium.

To derive the equilibrium we must endogenize the punishment $M$. From Lemma 2, we can see that we only need to solve equation (4) for $\underline{D}^{*}$ to characterize the strategies. The punishment is the expected profit lost by being held down to the one-shot profits $\pi_{o}$ in all future periods:

$$
M=\alpha \frac{\delta}{1-\delta} \int_{0}^{\bar{D}}\left(\pi_{c}(D)-\pi_{o}(D)\right) d F(D)=\frac{\gamma}{1-\gamma} \int_{0}^{\bar{D}}\left(\pi_{c}(D)-\pi_{o}(D)\right) d F(D)
$$

Here we have written $\gamma=\alpha \delta /(1-\delta+\alpha \delta)$. The collusive profits $\pi_{c}$ are determined as described above: pricing at the price cap for high and low demand realizations and otherwise at the maximum possible price that restricts the deviation gain enough. The most-collusive equilibrium will be the highest solution of $W\left(\underline{D}^{*}\right)=M\left(\underline{D}^{*}\right)$, since the firms will collude up to this point (and above the corresponding $\bar{D}^{*}$ ), with $\underline{D}^{*} \in[0, k]$. The threshold $\underline{D}^{*}$ hence determines the size of the expected punishment $M$. The equation is thus:

$$
\begin{aligned}
\underline{D}^{*} \bar{P} / 2= & \frac{\gamma}{1-\gamma}\left[\int_{0}^{\underline{D}^{*}} D \bar{P} / 2 d F(D)+\left[F(k)-F\left(\underline{D}^{*}\right)\right] \underline{D}^{*} \bar{P} / 2+\int_{k}^{\hat{D}}[D P(D) / 2-(D-k) \bar{P}] d F(D)\right. \\
& \left.+\int_{\check{D}}^{\bar{D}^{*}}[D P(D) / 2-(D-k) \bar{P}] d F(D)+\int_{\bar{D}^{*}}^{\bar{D}}[(k-D / 2) \bar{P}] d F(D)\right],
\end{aligned}
$$


where $\bar{D}^{*}, \hat{D}$ and $\check{D}$ depend on $\underline{D}^{*}$. The equation admits in general no closed-form solution, but can be solved numerically for standard demand distributions. However, the equation may have multiple solutions on $[0, k]$ : clearly, there is always one at zero, corresponding to the repeated non-collusive strategies. The most-collusive equilibrium derives from the highest of these. If the difference $W-M$ is increasing in $\underline{D}^{*}$ at the highest fixed point, collusion is not sustainable with larger $\underline{D}^{*}$. On the other hand, if the difference is decreasing, collusion with monopoly prices will be sustainable at $k$ as well, which makes it the most-collusive equilibrium choice. The following Proposition summarizes conditions for the existence of a collusive equilibrium.

Proposition 1. (a) Let $E_{G}=\int_{0}^{k} D / 2 d F(D)+\int_{k}^{\bar{D}}(k-D / 2) d F(D)$, and $\gamma_{H}=(1-\gamma) / \gamma$. If $E_{G} \geq$ $k \gamma_{H} / 2$, the firms collude with monopoly prices at all demand realizations.

(b) If the above condition does not hold, a sufficient condition for the existence of a collusive equilibrium (with collusive prices sometimes lower than $\bar{P}$ ) is $F(k) \geq \gamma_{H}$.

The firms' ability to sustain collusive prices depends crucially on both the distribution of demand and production capacities. Collusive prices can be sustained for all demand realizations when the expected deviation gain is large enough compared to the maximum deviation gain. Intuitively, with variable demand, full collusion becomes harder to sustain. Failing this, a "partially" collusive solution is sustainable when the fraction of demand below $k$ is high enough, or demand is low often enough. That is, overcapacity allows collusion to happen more often. This is similar to the results of Davidson and Deneckere (1990), who show that excess capacity is beneficial to collusion through higher threat of punishment, but in a model with constant demand.

Here, in conjunction with capacity, the distribution of demand affects the extent of collusion. When demand is concentrated on low (high) realizations, punishment is intuitively more effective for small (large) $k$. Collusion is thus generally easier to sustain with low $k$ for decreasing density functions, and with high $k$ for increasing densities, but the impact of $k$ may be non-monotonic for general distributions. Figure 2 shows pricing patterns for different $k$ for two distributions: uniform and a decreasing PDF. (Note that for the given model parameters, the range $[\hat{D}, \check{D}]=\emptyset$, and the collusive equilibrium is always in pure strategies.) In the left panel, with a decreasing demand PDF, the impact of capacity on pricing depends on demand, but fully collusive prices can be sustained most often with low capacity, resulting in highest average prices. In the right panel, with a uniform density, the effect of $k$ on the extent of collusion is non-monotonic: collusive prices are on average highest with intermediate capacity, similarly to the results in Anderson and Cau (2011). An increasing PDF (not pictured) would imply fully collusive prices more often at high capacities.

To further illustrate this equilibrium, we present some preliminary evidence that prices set by peaking plants may take a shape similar to the one predicted by our model. Returning to the 
Figure 2 Collusive pricing for capacities different $k$ and demand distributions.

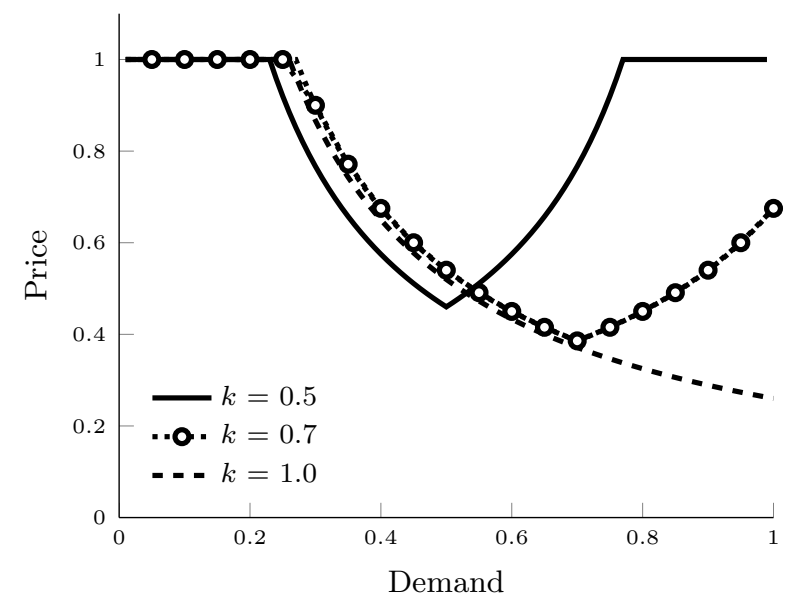

(a) Beta distribution $(1,3)$ (decreasing PDF)

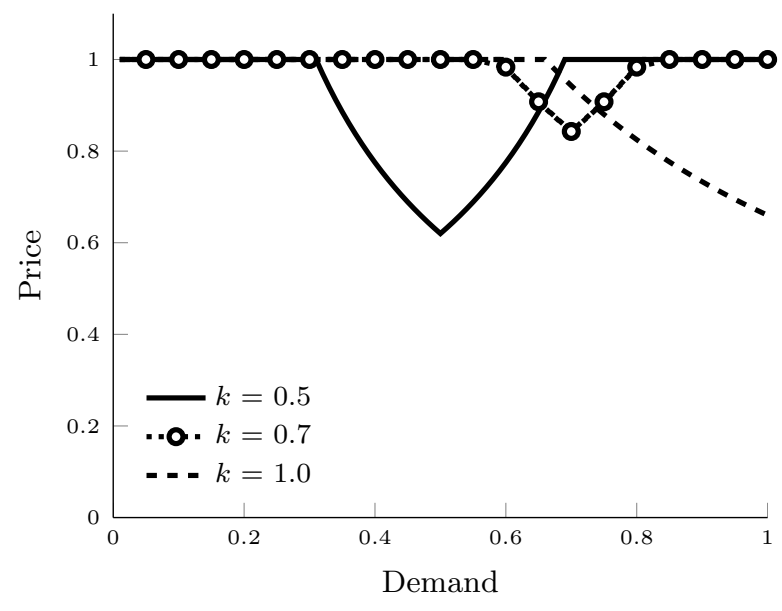

(b) Uniform distribution

Note. $\bar{P}=1, \bar{D}=1$ and $\gamma=0.6$.

Figure 3 Average price for which oil plants sold electricity in July 2006 in the UK market as a function of fraction of excess capacity available from other fuel types, with two standard error bands.

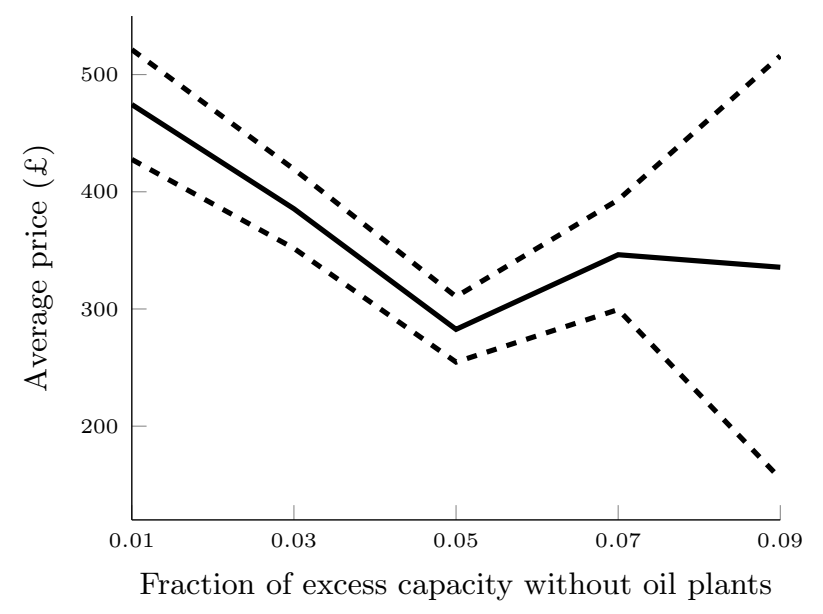

example of the British market in July 2006, Figure 3 presents the average price for which oil plants sold their power to the balancing market in that period, compared to the fraction of available capacity (reserve margin) in the market without these plants. The average price achieved by oil peakers appears to be highest with highest scarcity, but to reach its minimum with mid-level demand. However, the standard errors of the estimates are too large to provide conclusive evidence for this. Whilst there are a number of other factors influencing pricing behaviour, such as portfolio considerations (see Section 4), the figure illustrates the plausibility of our equilibrium result.

\section{Revenue Targets}

We now introduce performance targets for the generators, representing financial objectives under which they operate. Such targets may exist for two reasons. First, investors or lenders may penalize 
a company if a plant is not profitable over a time period, that is, unable to at least cover its longrun fixed costs. In the extreme, financial under-performance may force a (potentially long-term profitable) plant to be retired from the market, reducing the total capacity available to meet peak demand. In particular, weak energy demand and increasing low marginal cost renewable production have caused such developments in many countries (e.g., PR Newswire 2004, Eckert 2013).

Second, even without any threat of plant retirement, revenue targets may be used by stakeholders to provide managerial incentives. For example, the manager of a plant might be expected to achieve at least a certain rate of return on investment, with compensation tied to this target. As the role of peaking plants is expected to increase with more intermittent generation entering the technology mix, managerial incentives to operate these assets most effectively will increase and the question therefore arises whether the use of targets should be pro-actively developed for profit maximisation. From our analysis, it seems that it should.

Below, we show how revenue targets link pricing decisions to the state of accumulated revenues as well as time remaining before the target period. Neither the non-collusive nor the collusive game is stationary in this case. The collusive game, beyond the impact of targets on deviation incentives, is affected by the dynamics of the non-collusive game (as punishment), which can be sharply different. We consider two possible consequences of missing a target: financial penalties and plant closure or mothballing. First, we assume that there is no threat of plant closure, but let the producers face a financial penalty function, which may represent either managerial incentives or, more generally, any penalty faced by the plant for not meeting the target, such as increased cost of credit, impairment costs, or downgrades. We derive both the non-collusive and collusive equilibria associated with a penalty and illustrate their differences. The impact of targets for collusive prices is ambiguous and non-monotonic as they affect both deviation (to increase chance of reaching the target) and punishment (to reduce this chance) incentives. For non-collusive prices, the impact is also non-monotonic, but often qualitatively in different direction than for collusive prices. Second, we allow a plant not meeting its target to be mothballed or retired from the market with some probability. In addition to reaching its own target, a producer then has an incentive to reduce the chance of the other producer meeting its target to remain in the market as a monopolist. Hence, collusive prices are less likely to be sustained.

Let us introduce the model with financial penalties. The plants are faced with revenue targets $\Pi^{i}$ with $\Pi=\left(\Pi^{1}, \Pi^{2}\right)$, at a common time $T$ (for example, the end of a financial year). The targets and penalties faced by each firm are common knowledge from the beginning of the horizon: there is no asymmetric information in the game. After $T$, the game continues as the stationary game above. (We discuss multiple targets below.) For firm $i$ at period $\tau$, expected utility depends on 
demand uncertainty but also on the pricing decisions made by both plants in the future and thus their respective revenue states:

$$
\mathbb{E}\left(u^{i}\left(\tau, \Pi_{\tau}\right)\right)=\mathbb{E}\left(\sum_{t=\tau}^{\infty} \delta^{t-\tau} \pi_{t}^{i}\right)-\delta^{T-\tau} \mathbb{E}\left[C\left(\Pi_{\tau}^{i}-\sum_{t=\tau}^{T} \pi_{t}^{i}\right)^{+}\right],
$$

where $\sum_{t=\tau}^{\infty} \delta^{t-\tau} \pi_{t}^{i}$ is the sum of discounted period revenues $\pi_{t}^{i}, C(x)^{+}=C(\max (x, 0))$ is a penalty function for a shortfall of $x$ at time $T$ and $\Pi_{\tau}^{i}=\Pi^{i}-\sum_{t=1}^{\tau-1} \pi_{t}^{i}$ is the current period revenue target. Typically we would expect the function $C(x)^{+}$to be concave (when $x \geq 0$ ): missing the target matters more than the amount of shortfall. In the extreme, Levin et al. (2008) use a constant discrete penalty to capture this. We require continuity of the function for equilibrium existence in the capacitated case $\bar{D}>k$ (our formulation can closely approximate a step penalty with a suitably concave functional form). In the uncapacitated case, we can allow discontinuities. Notice that a concave penalty function implies that far enough from the target, the producers are riskseeking (approaching the target is very valuable). But at the target this does not hold as there is no additional gain for going beyond it. Hence, the target causes an "inflection point" in utility. A similar utility function can be found in e.g. Rochet and Villeneuve (2011).

\subsection{Non-collusive pricing}

Let us first analyse how revenue targets influence non-collusive pricing. Above, the non-collusive equilibrium was stationary, with marginal cost pricing for low demand and mixed strategies for high demand. Now the potential penalty changes pricing incentives over time depending on accumulated revenues. With low demand $(D \leq k)$, revenue targets reinforce undercutting incentives and marginal cost pricing is still the unique equilibrium. When $D>k$, pricing becomes dynamic. Consider the final period before the target, with the targets of the firms are $\Pi_{T}^{1} \geq \Pi_{T}^{2}$ and penalty function $C\left(\Pi_{T}^{i}-x\right)^{+}$. The profits for being a high or low bidder are

$$
\begin{aligned}
& u^{i, h}(T, p)=p(D-k)-C\left(\Pi_{T}^{i}-p(D-k)\right)^{+} ; \\
& u^{i, l}(T, p)=p k-C\left(\Pi_{T}^{i}-p k\right)^{+} .
\end{aligned}
$$

A pure strategy equilibrium cannot exist, so we consider mixed strategies with CDF's $H_{T}^{i}(p)$. Setting the expected profits equal for all $p$, we have:

$$
u^{i}(T, p)=H_{T, n c}^{j}(p)\left[p(D-k)-C\left(\Pi_{T}^{i}-p(D-k)\right)^{+}\right]+\left(1-H_{T, n c}^{j}(p)\right)\left[p k-C\left(\Pi_{T}^{i}-p k\right)^{+}\right] .
$$

The resulting mixed strategy equilibrium can be extended recursively to earlier periods.

Proposition 2. In the dynamic game with revenue targets at time $T$ and penalty functions $C(x)^{+}$, there is a unique non-collusive equilibrium: 
1. If $D \leq k, p=0 \forall i$.

2. If $k<D \leq 2 k$, the equilibrium is in mixed strategies defined recursively through

$$
\begin{aligned}
H_{t, n c}^{i}(p) & =\frac{p k+\delta \mathbb{E}\left[u^{j}\left(t+1, \Pi_{t}^{j}-p k\right)\right]-\bar{P}(D-k)-\delta \mathbb{E}\left[u^{j}\left(t+1, \Pi_{t}^{j}-\bar{P}(D-k)\right)\right]}{p(2 k-D)-\delta \mathbb{E}\left[u^{j}\left(t+1, \Pi_{t}^{j}-p(D-k)\right)\right]+\delta \mathbb{E}\left[u^{j}\left(t+1, \Pi_{t}^{j}-p k\right)\right]}, \quad t<T, \\
H_{T, n c}^{i}(p) & =\frac{p k-C\left(\Pi_{T}^{j}-p k\right)^{+}-\bar{P}(D-k)+C\left(\Pi_{T}^{j}-\bar{P}(D-k)\right)^{+}}{p(2 k-D)+C\left(\Pi_{T}^{j}-p(D-k)\right)^{+}-C\left(\Pi_{T}^{j}-p k\right)^{+}},
\end{aligned}
$$

with $p \in[\bar{P}(D-k) / k, \bar{P}]$. The utilities are updated according to

$$
\begin{aligned}
& u_{t, n c}^{i}\left(\Pi_{\tau+1}\right)=\mathbb{E}\left[\bar{P}(D-k)^{+}+\delta u_{t+1, n c}^{i}\left(\Pi_{t+1}\left(\Pi_{t},(D-k)^{+}, \bar{P}\right)\right)\right] \\
& u_{T+1, n c}^{i}\left(\Pi_{T+1}^{i}\right)=\sum_{\tau=1}^{\infty} \delta^{\tau} \mathbb{E}\left[\pi_{o}(D)\right]-C\left(\Pi_{T+1}\right)^{+}
\end{aligned}
$$

Revenue targets affect mixed strategies through three terms; note that the target of firm $j$ affects the pricing of firm $i$. Consider last period prices: when $\Pi_{T}^{j}$ is low and all the penalty function terms are zero, the mixed strategy distribution corresponds to the stationary (risk-neutral) distribution. With a higher $\Pi_{T}^{j}$, only $C\left(\Pi_{T}^{j}-p(D-k)\right)^{+}$is positive, and a penalty is only incurred if the firm is the high bidder. To keep the expected utility constant, prices then need to shift up to compensate for this. However, when $\Pi_{T}^{j}$ further increases so that $C\left(\Pi_{T}^{j}-\bar{P}(D-k)\right)^{+}$is also positive, the total impact is no longer clear: the penalty now results in a decrease in expected utility and hence potentially lower prices. Finally, when $C\left(\Pi_{T}^{j}-p k\right)^{+}$is also positive, a low-pricing firm is also penalised; however, this penalty is always lower than that of the residual-producing firm at the same offer price. The overall effect then depends on $C(x)$ : if it is convex, the prices will be higher; if concave, they will be lower. The shape of the mixed strategy distribution is summarized as follows.

Proposition 3. Compared to the one-shot mixed equilibrium offer price distribution $H_{o}^{i}$, in the dynamic non-collusive equilibrium in period $T$,

1. When $\Pi_{T}^{j} \leq p(D-k), H_{T, n c}^{i}(p)=H_{o}^{i}(p)$.

2. When $p(D-k)<\Pi_{T}^{j}<\bar{P}(D-k), H_{T, n c}^{i}(p)<H_{o}^{i}(p)$. The difference $H_{o}^{i}(p)-H_{T, n c}^{i}(p)$ is highest when $p(D-k) \rightarrow \Pi_{T}^{j}$.

3. When $\bar{P}(D-k) \leq \Pi_{T}^{j} \leq p k, H_{T, n c}^{i}(p) \leq H_{o}^{i}(p)$ if $C(x)$ is convex; if it is concave, we may have $H_{T, n c}^{i}(p)>H_{o}^{i}(p)$.

4. When $p k<\Pi_{T}^{j}, H_{T, n c}^{i}(p) \leq H_{o}^{i}(p)$ iff $C(x)$ is convex.

Here $H_{T, n c}^{i}(p) \leq H_{o}^{i}(p)$ implies prices concentrated on higher values with the target. The overall effect on average prices is a combination of the above effects. Unfortunately similar properties are not available in earlier periods as it is in general not possible to guarantee the convexity or concavity of $\mathbb{E}\left[u^{i}\right]$. However, the impact on prices is typically strongest in the first period and is 
Figure 4 Dynamic non-collusive prices: mean price over all $D$ given distance to target at different $T$.

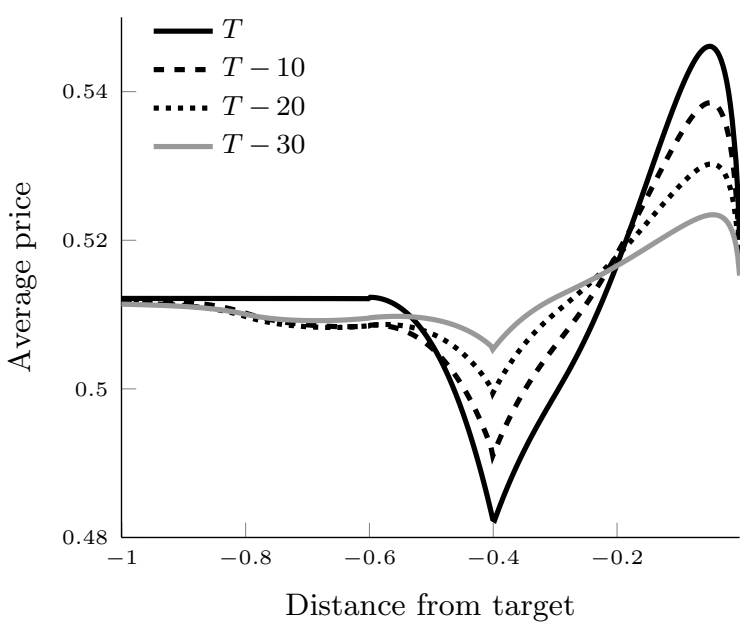

Note. $\bar{P}=1, \bar{D}=1, \delta=0.96, \alpha=0.07, C=R_{c} x^{0.001}$, uniform demand.

mitigated over time: as the horizon increases, the penalty becomes irrelevant and the distribution tends to the static distribution (as long as the penalty function is concave).

We can interpret the pricing impacts in terms of risk preferences. As noted above, with a concave penalty, the producers are effectively risk-averse close to the target and risk-seeking further from it. In both cases, mixing distributions need to keep expected utility constant at any offer price. Here, going far beyond the target does not matter. To compensate for this, there is a positive premium in prices. Conversely, in the risk-seeking case, there is a negative premium as approaching the target is disproportionately valuable. Figure 4 illustrates the impact on average prices over time at different shortfalls to the target revenue (which are the same for both firms), as well as prices for a particular demand realization. The figure shows a significant impact on average prices; on particular demand instances, the effect can be very sharp, increasing the mean price of the mixing distribution by more than $100 \%$.

\subsection{Collusive pricing}

The dynamic non-collusive equilibrium established above also serves as a punishment equilibrium for collusive strategies. Let us now derive the most-collusive strategies in the dynamic game. We focus on the case where at least one of the generators is below its revenue target; otherwise the game is exactly like the one described in Section 2. We can write the game recursively, with a final payoff based on the continuation value after time $T$ when the problem shifts from a statedependent dynamic game to the stationary repeated one. Following the construction of most- 
collusive strategies in the stationary case, we derive the highest collusive price $p_{c}$ that the producers can sustain:

$$
\begin{aligned}
& p_{c}=\min _{i} \arg \max _{p\left(\tau, D, \Pi_{\tau}\right)} \mathbb{E}\left[u^{i}\left(\tau, D, \Pi_{\tau}, p\right)\right] \\
& \mathbb{E}\left[u^{i}\left(\tau, D, \Pi_{\tau}, p\right)\right]=D p / 2+\delta \mathbb{E}\left[u^{i}\left(\tau+1, \Pi_{\tau+1}\left(\Pi_{\tau}, D / 2, p\right)\right)\right], \forall i \\
& u^{i}\left(T+1, D, \Pi_{T+1}\right)=R_{c}-C\left(\Pi_{T+1}^{i}\right)^{+} .
\end{aligned}
$$

Here $R_{c}=\sum_{\tau=1}^{\infty} \delta^{\tau} \mathbb{E}\left[\pi_{c}(D)\right]$ is the expected stationary collusive revenue after time $T$. The highest price is the minimum of the two firms' prices (we show that this is optimal below). Expected utility of the firms with this collusive price is updated through equations (17)-(18). The prices are sustainable if deviations are not profitable:

$$
\begin{aligned}
& \mathbb{E}\left[u^{i}\left(\tau, D, \Pi_{\tau}, p\right)\right] \geq \mathbb{E}\left[u_{d e v}^{i}\left(\tau, D, \Pi_{\tau}, p\right)\right] \forall i, \\
& \mathbb{E}\left[u_{d e v}^{i}\left(\tau, D, \Pi_{\tau}, p\right)\right]=\min \{k, D\} p+\delta u_{t, n c}^{i}\left(\Pi_{\tau+1}\left(\Pi_{\tau}, \min \{k, D\}, p\right)\right) \\
& u_{t, n c}^{i}\left(\Pi_{\tau+1}\right)=\mathbb{E}\left[\bar{P}(D-k)^{+}+\delta u_{t+1, n c}^{i}\left(\Pi_{t+1}\left(\Pi_{t},(D-k)^{+}, \bar{P}\right)\right)\right] \\
& u_{T+1, n c}^{i}\left(\Pi_{T+1}^{i}\right)=R_{n c}-C\left(\Pi_{T+1}\right)^{+},
\end{aligned}
$$

where $R_{n c}=\sum_{\tau=1}^{\infty} \delta^{\tau} \mathbb{E}\left[\pi_{o}(D)\right]$ is the non-collusive expected discounted revenue after time $T$. The expected deviation profit (20) includes profit in the current period as well as future profits from the dynamic non-collusive equilibrium derived above, updated through eqs. (21)-(22).

When capacity is constrained, producers may prefer mixed strategies to the above collusive prices. Notice that this equilibrium is different from the non-collusive equilibrium above as future periods are collusive. As these profits depend on both players' distances from targets and future collusive profits, the mixed strategies need to be solved simultaneously with the rest of the collusive equilibrium. We derive the optimal strategies and the profits in this "mixed collusive" equilibrium in the e-companion. In sum, if deviating to the price cap is preferred by either of the firms, mixed strategies are played instead. Then profits are the mixed strategy profits, which need to equate expected utility with this deviation. Otherwise the collusive price is equal to $p_{c}$, the highest price at which both firms can sustain collusion. To account for the collusive mixed strategies, we thus need to check whether either of the firms would deviate from the highest collusive price (24):

$$
\begin{aligned}
& \mathbb{E}\left[u^{i}\left(\tau, D, \Pi_{\tau}\right)\right]=\left\{\begin{array}{ll}
D p_{c} / 2+\delta \mathbb{E}\left[u^{i}\left(\tau+1, \Pi_{\tau+1}\left(\Pi_{\tau}, D / 2, p_{c}\right)\right)\right] & \text { if } \max _{i} \zeta^{i}=0 \\
(D-k)^{+} \bar{P}+\delta \mathbb{E}\left[u^{i}\left(\tau+1, \Pi_{\tau+1, M}\left(\Pi_{\tau}\right)\right)\right] & \text { otherwise }
\end{array} \quad \tau \in\{1, \ldots, T\}\right. \\
& \zeta^{i}\left(\tau, D, \Pi_{\tau}\right)=\mathbb{1}\left(D p_{c} / 2+\delta \mathbb{E}\left[u^{i}\left(\tau+1, \Pi_{\tau+1}\left(\Pi_{\tau}, D / 2, p_{c}\right)\right)\right] \leq\right. \\
&\left.(D-k)^{+} \bar{P}+\delta \mathbb{E}\left[u^{i}\left(\tau+1, \Pi_{\tau+1, M}\left(\Pi_{\tau}\right)\right)\right]\right), \forall i .
\end{aligned}
$$


Here $\mathbb{1}(\cdot)$ denotes the indicator function and $\zeta^{i}=1$ signifies deviation from collusive prices, and hence mixed strategies. When $\max _{i} \zeta^{i}=1$, that is, at least one firm prefers deviating, mixed strategies are played. Moreover, $\Pi_{\tau}=\left(\Pi_{\tau}^{i}, \Pi_{\tau}^{j}\right)$ denotes the state of the game, updated as $\Pi_{\tau+1}\left(\Pi_{\tau}, x, p\right)=$ $\left(\Pi_{\tau}^{i}-x p, \Pi_{\tau}^{j}-(D-x) p\right)$. With mixed strategies, the update is $\Pi_{\tau+1, M}\left(\Pi_{\tau}\right)=\left(\Pi_{\tau}^{i}-\bar{P}(D-k)^{+}, \Pi_{\tau}^{j}-\right.$ $\left.\bar{P}(D-k)^{+2} / k\right)$.

The equilibrium is summarized in the following Proposition.

Proposition 4. The most-collusive price of the dynamic problem with revenue targets is found through problem (16)-(24). There may be either more or less collusion before the revenue target period than in the base-case model. That is, letting $0<p_{b}<\bar{P}$ be the stationary average collusive price, $\exists \tau_{l}, \Pi_{l, \tau}$ s.t. $p_{c}\left(\tau_{l}, \Pi_{l, \tau}\right)<p_{b}$. If further $k$ is high enough, $\exists \tau_{h}, \Pi_{h, \tau}$ s.t. $p_{c}\left(\tau_{h}, \Pi_{h, \tau}\right)>p_{b}$.

The impact of revenue targets on collusive prices is ambiguous and non-monotonic. In contrast to Section 2, we can no longer assume that deviation gains are monotone in demand for $D \leq k$ and $D>k$. Consequently, the structure of the base-case solution (monopoly pricing for low/high demand and lower prices closer to $k$ ) does not carry through in general.

Uncapacitated game. To clearly lay out the pricing incentives, we shall first go through the intuition in the uncapacitated version of the problem $(\bar{D} \leq k)$. Consider period $T$, and suppose the shortfall to the target revenue is the same for both plants, and relatively small. Collusive prices are set low enough to just discourage deviation. This is illustrated in Figure 5(a) for a step penalty function. In the stationary game, prices would be constant at $\bar{P}$ until $\underline{D}^{*}$, and then decreasing. With targets, deviating to reach the target would be profitable at $D \in[0.1,0.2)$, where the target can be reached by deviating but not by colluding. The prices are hence adjusted down in this range, but otherwise the strategies are unchanged.

More generally, revenue targets induce non-monotonic dynamic prices. With a small distance to the target, deviation gains are still increased, but punishment is also affected. With $\bar{D} \leq k$, a deviating plant makes zero profits in the punishment equilibrium and hence will not further approach the target. This strengthens the punishment; however, the punishment may also decrease as later collusive profits may be lower closer to the target due to higher deviation incentives. In sum, prices tend to be low when the shortfall is fairly small (due to lucrative deviations), high when the shortfall increases (due to more severe punishment) and not affected when it is further increased as incentives return to the stationary level. This pattern can be found in Figure 5(b), which displays mean prices achieved by the plants given their (equal) shortfall to the target and the (equal) time left before the target period, with a step penalty function. Both distance and time left before the target considerably affect pricing, yet the overall pricing impact is not obvious due to the conflated changes in deviation gains and punishment. 
Figure 5 Prices for particular distance 0.1 from the target at time $T$ (a), and mean prices as a function of distance and time to revenue target (b), with no capacity constraints.

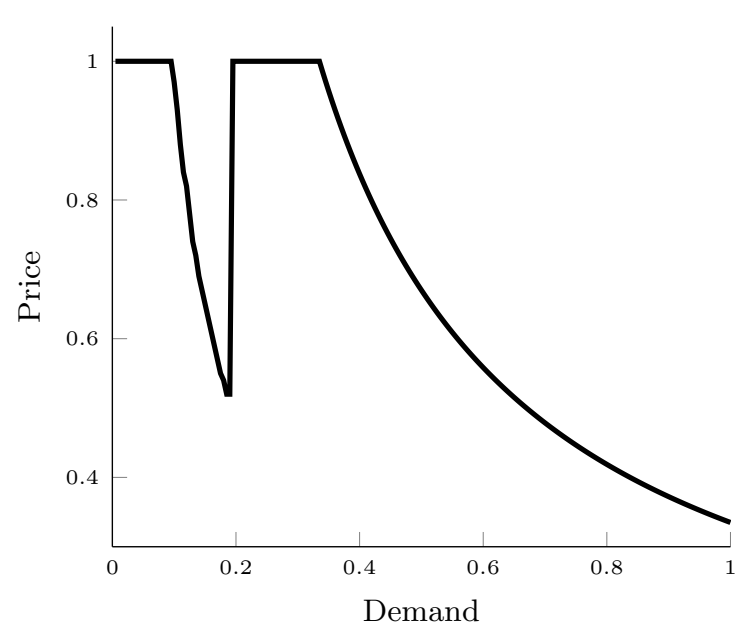

(a)

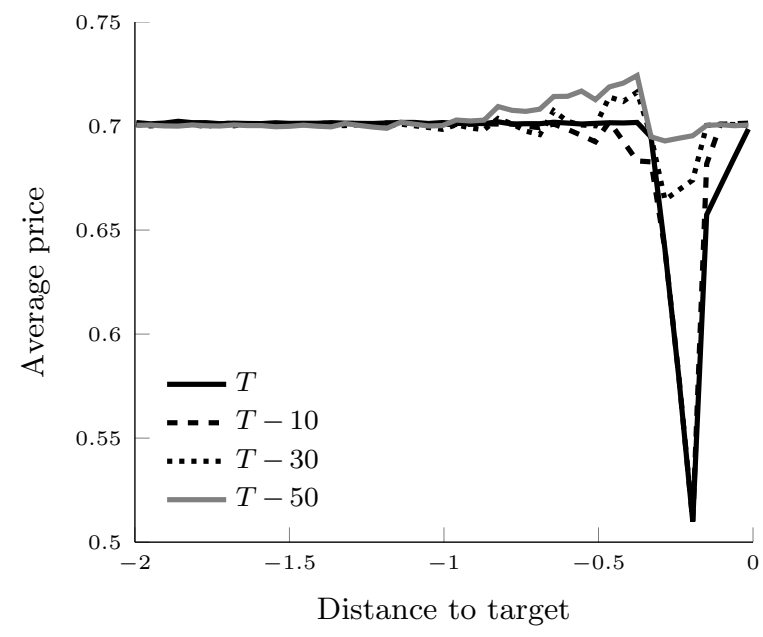

(b)

Note. $\bar{P}=1, \bar{D}=1, \alpha=0.05, \delta=0.96, C=R_{c}$, uniform demand.

These effects are, surprisingly, often qualitatively opposite to those in the (capacity-constrained) non-collusive equilibrium. There, the mixed strategies aim keep the competitor's expected profits constant. Hence they change reflecting the chance of meeting the target: close to it, going far beyond the target adds little value and hence prices are on average higher ("risk aversion"). Further away, the opposite is true ("risk seeking"). With collusion, by contrast, the determining factor is balancing (the most binding) deviation gains and punishment. Near the target, meeting it matters more than what happens beyond, and the increased deviation gains lower prices. Further away, missing the target is more likely after deviating, and higher punishment increases prices. A risk interpretation is not straightforward in general, but to a certain extent, we can interpret the incentive to deviate to reach the target as a risk-averse action. Conversely, the increased punishment is closely related to the disproportionate value of closing the gap ("risk seeking").

Capacitated game. Let us now discuss the impact of capacity constraints $(\bar{D}>k)$ on pricing. The above-discussed deviation and punishment incentives persist; however, punishment can no longer guarantee not reaching a target due to the positive non-collusive markups in the punishment equilibrium. The dynamic equilibrium further often involves mixed strategies being played as part of it; therefore, even if the producers start at equal targets, they are likely to end up at different distances, and asymmetries in targets hence become more relevant. With unequal distances, prices decrease: the producers set the highest price both can support. In general, the relationship between prices and demand is complex and non-monotonic. With a concave penalty approximating a step function, prices may again exhibit jumps to adjust for reaching the target. 
Figure 6 Prices for particular distances from the target (a) and mean prices as a function of distance and time to revenue target (b), constrained capacity.

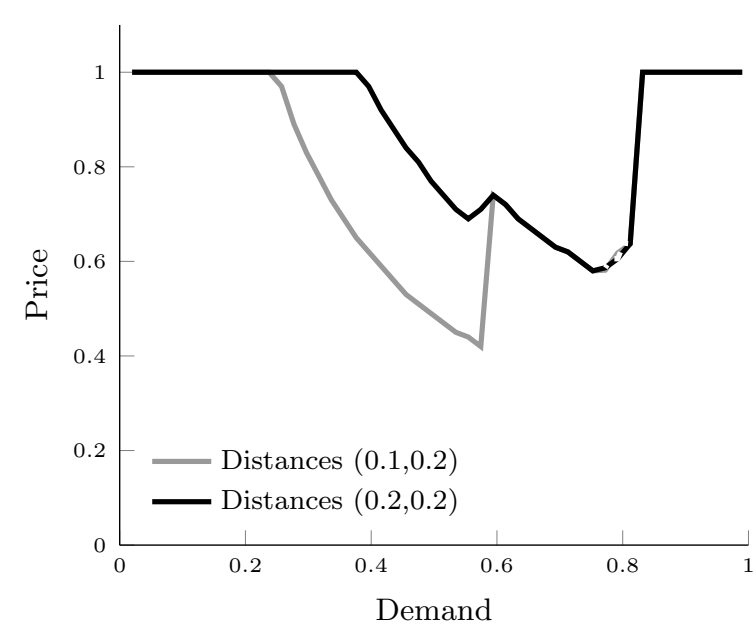

(a)

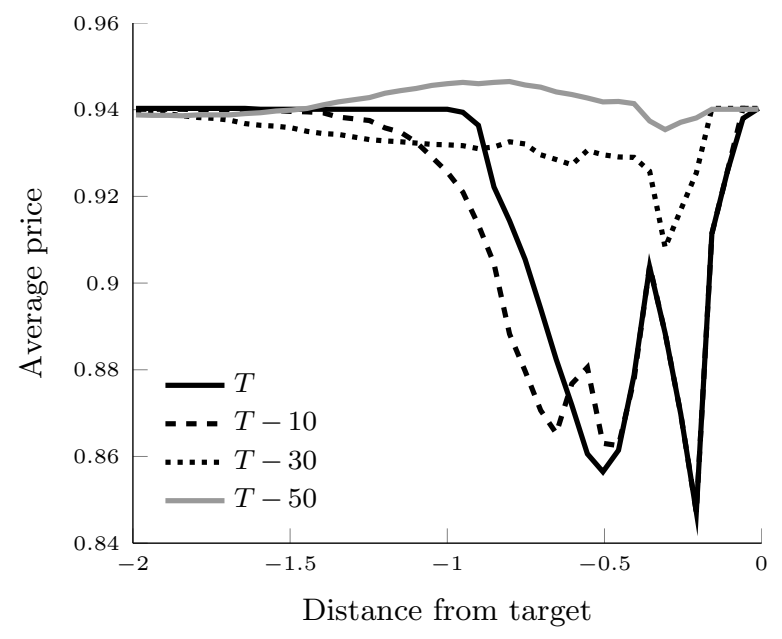

(b)

Note. $\bar{P}=1, \bar{D}=1, \mathrm{k}=0.55, \alpha=0.06, \delta=0.96, C(x)=R_{c} x^{0.001}$, uniform demand.

These effects are illustrated in Figure 6(a), with two pairs of distances pictured at $T$. With equal distances, prices are equal to $\bar{P}$ when demand is low enough so that the target cannot be reached. Prices then decrease to control deviation incentives, and increase above $k$. With higher demand, collusive prices again decrease: demand increases so pre-empting deviations to reach the target requires lower prices. Prices in fact decrease enough that mixed strategies are used along the dotted line around $D=0.8$. Finally prices jump up to $\bar{P}$ with sufficiently high demand when deviation to supply full demand is no longer profitable. With unequal distances, prices are never higher; they are lower for intermediate demand values as the producer closer to its target has higher incentives to deviate.

Again, the impact on collusive prices of the target is often qualitatively the opposite of that in the non-collusive equilibrium. As non-collusive prices constitute the punishment for deviating, this effect further serves to reduce prices in the collusive equilibrium. Close to the target, prices again decrease in late periods, but may still increase in earlier periods due to the punishment effect, even with tight capacity constraints. Recall that in Section 2, we noted the impact of excess capacity on collusive prices. Revenue targets exacerbate this impact: with loose capacity constraints, prices can increase more as the target can be used more directly as additional punishment. These effects are illustrated in Figure 6(b) for fairly constrained capacity: the negative effects of targets dominate especially in periods close to the target. The general impact of targets with capacity constraints is complex, resulting from the dynamics of the above incentives, but also the concurrent dynamics of the punishment equilibrium. 
Equal targets. In the above examples, we have focused on the case where the plants start with equal revenue targets. In fact, it turns out that if they are able to commit to targets, this is indeed optimal. The intuition is simple: collusive prices are not increased by asymmetry, so different targets tend to result in lower prices. Furthermore, given the prospect of higher prices, imposing strictly positive risk constraints to their plants may increase the expected profits of risk-neutral (owners of) firms.

Proposition 5. Consider problem (16)-(24). Before the price game, it is optimal for the plants to set equal revenue targets $\Pi^{1}=\Pi^{2}=\Pi^{*} \geq 0$.

Multiple targets. Instead of a single target, producers are likely to face several (say yearly) revenue targets in succession. Adding multiple targets to the horizon does not significantly change our qualitative conclusions. Suppose that there is a revenue target at every $T$ periods. Then the problem is stationary in that during each cycle of length $T$, the optimal strategies will be exactly the same. The conclusion is that since profits in future cycles are treated in a risk-neutral way, having multiple revenue targets may further increase the prices that the plants can achieve. Essentially, if the targets are set in such a way that expected profits increase, the punishment becomes higher, which further decreases deviation incentives (assuming commitment to these targets). With many repetitions, the marginal impact declines and prices converge.

Proposition 6. Consider problem (16)-(24). With multiple targets, prices are increased in expectation, compared to the single target case, if and only if the punishment at the beginning of each target horizon is higher than the punishment in the stationary game.

Frequency of interaction. In the stationary model, we were able to combine the discount factor $\delta$ and the probability of interaction $\alpha$ into a single parameter $\gamma$, but with revenue targets this is no longer true as the game is dynamic. We can directly translate the number of periods left into the number of remaining demand events, which follows the binomial distribution $\operatorname{Bin}(\alpha, T-t)$ with mean $\alpha(T-t)$. To compare the relative impacts of the two parameters, we hold $\gamma$ constant and vary $\delta$ :

$$
\alpha=\frac{\gamma}{1-\gamma} \frac{1-\delta}{\delta}, \quad \delta \in[\gamma, 1]
$$

where $\delta$ is constrained so that $\alpha \in[0,1]$. Holding $\gamma$ constant, prices beyond the target remain the same as we vary $(\delta, \alpha)$. We can thus distinguish the impact of the frequency of interaction when holding the baseline collusive profits constant (if it is increased holding $\delta$ constant, prices increase).

The frequency of interaction affects future payoffs in several ways. A high frequency guarantees more opportunities to reach the target in both collusive and punishment equilibria, and punishment 
may hence become either more or less effective. At the same time, a high frequency corresponds to less weight on the future and hence lower expected punishment. The pricing intuition is illustrated in Figure 7, where average prices for different periods and parameter combinations are displayed. As above, highest prices are achieved at "moderate" distances, and with enough time left before the target, when punishment becomes more important than deviation gain. With frequent interactions, this happens at earlier periods (as punishment starts to matter) and at higher shortfall from the target (as revenues accumulate more). Punishment is high with many opportunities to reach the target, but deviation would still radically decrease the chance of meeting the target. However, when the shortfall is increased, prices again decrease below the stationary level, as collusive prices reached tend to be lower in the remaining periods before the target (panels a and b). As time left before the target is increased, incentives are moderated by both discounting and more certainty in reaching the target. With infrequent interactions, price increases happen at later periods, but are discounted more slowly (panels c and d). Overall, the combination that yields highest prices crucially depends on both the distance and time left to the target. Timing and magnitude of targets hence matter for pricing outcomes depending on the expected number of interactions left.

\subsection{Plant retirement}

We shall now allow the plants to be mothballed or retired after failing to meet the revenue target. This creates the additional incentive to not only meet the target to continue in the market but also to become a monopolist by forcing its competitor not reach its target.

Let us denote the decision to retire a plant (or alternatively mothball it indefinitely) after the target period as $\mu^{i} \in\{0,1\}$, where 1 stands for retirement. The probability of retirement given distance to target is defined as

$$
\theta\left(\Pi^{i}\right)=\operatorname{Pr}\left(\mu^{i}=1 \mid \Pi^{i}\right)
$$

and is assumed to be exogenous and the same for both plants. We further assume the limits $\lim _{\Pi^{i} \uparrow \infty} \theta\left(\Pi^{i}\right)=\theta_{\infty}$ and $\lim _{\Pi^{i} \downarrow 0} \theta\left(\Pi^{i}\right)=\theta_{0}$, with $1 \geq \theta_{\infty} \geq \theta_{0} \geq 0$ and $\theta(x)$ (weakly) increasing in $x$ and concave to capture the marginal impact of missing the target. Finally, $\theta\left(\Pi^{i}\right)=0$ for $\Pi^{i} \leq 0$. In words, missing the target by a larger amount makes mothballing more likely, while meeting it removes this risk completely. For example, letting $\theta_{\infty}=1$ and making the function sufficiently concave, a plant not meeting its target will practically always be retired.

Continuation payoffs after the target change according to retirement decisions. A plant continuing alone will receive monopoly profits $\pi_{m}=\min \{D, k\} \bar{P}$ throughout, while both plants continuing together will act as in the model above, receiving the stationary collusive profits $\pi_{c}$. If a plant is retired, its profit is zero. (We assume full commitment to these decisions on the part of the owners 
Figure 7 Mean prices for different $(\delta, \alpha)$-pairs given (equal) shortfall to target and time to target period.

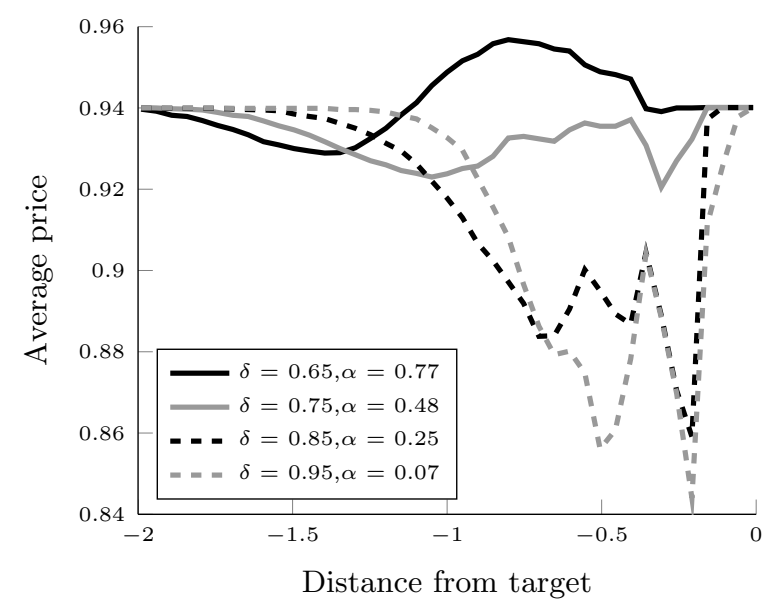

(a) $T-5$.

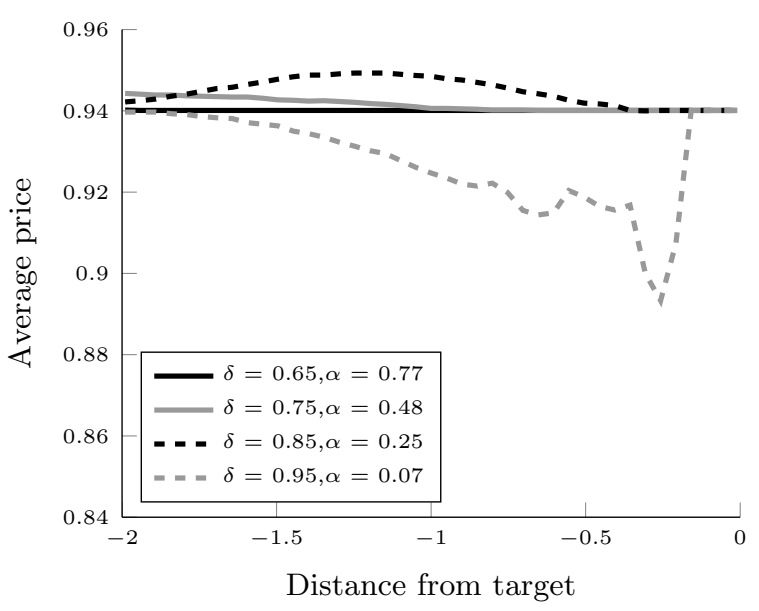

(c) $T-20$.

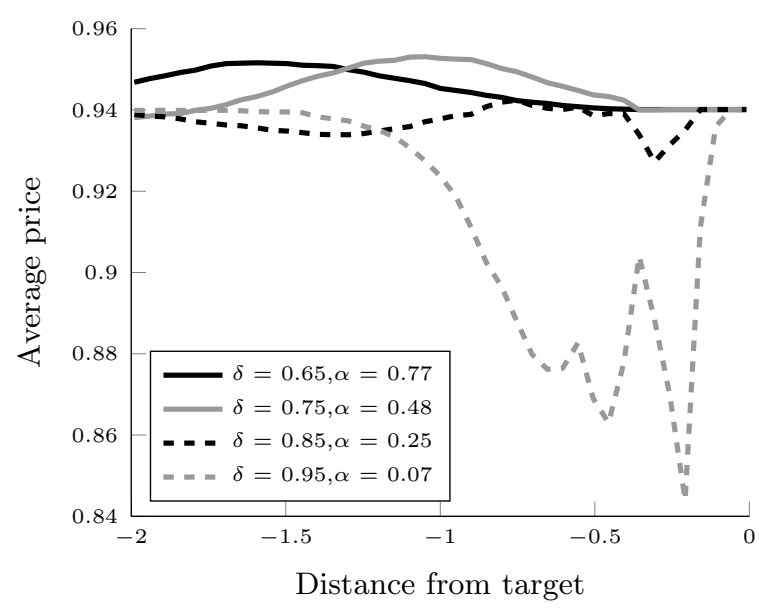

(b) $T-10$.

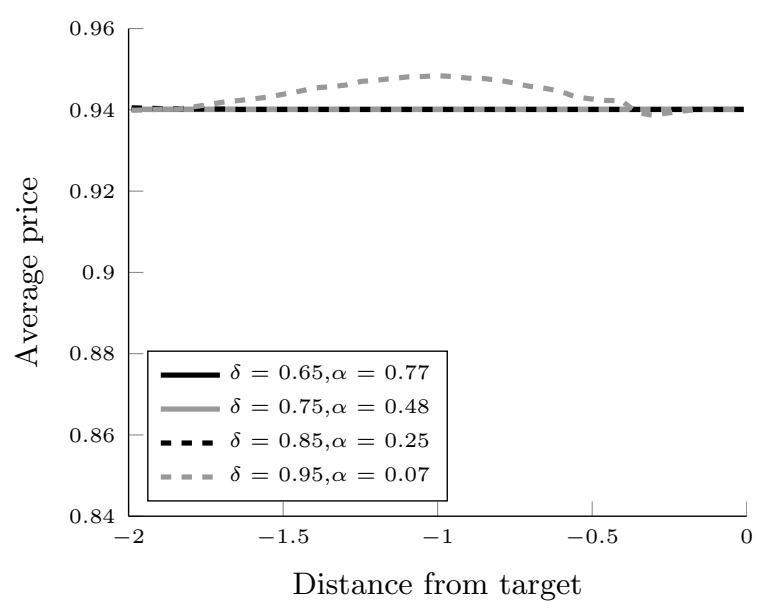

(d) $T-50$.

Note. $\bar{P}=1, \bar{D}=1, k=0.55, C(x)=R_{c} x^{0.001}$, uniform demand.

of the plant.) The modified incentives affect both non-collusive and collusive pricing. The final period continuation profits are changed to

$$
\begin{aligned}
R_{c, r e t}^{i}\left(\Pi_{T+1}\right) & =-C\left(\Pi_{T+1}^{i}\right)^{+}+\left(1-\theta\left(\Pi_{T+1}^{i}\right)\right)\left(\theta\left(\Pi_{T+1}^{j}\right) R_{m}+\left(1-\theta\left(\Pi_{T+1}^{j}\right)\right) R_{c}\right), \\
R_{n c, r e t}^{i}\left(\Pi_{T+1}\right) & =-C\left(\Pi_{T+1}^{i}\right)^{+}+\left(1-\theta\left(\Pi_{T+1}^{i}\right)\right)\left(\theta\left(\Pi_{T+1}^{j}\right) R_{m}+\left(1-\theta\left(\Pi_{T+1}^{j}\right)\right) R_{n c}\right) .
\end{aligned}
$$

Here $R_{k}=\sum_{\tau=1}^{\infty} \delta^{\tau} \mathbb{E}\left[\pi_{k}(D)\right]$ is the expected discounted sum of stationary profits in each scenario after the target, and $\Pi_{T+1}^{i}=\Pi_{T}^{i}-\pi_{T}^{i}$. We relegate the derivation of the equilibrium to the Electronic Companion; here we note that plant retirement poses some technical constraints on equilibrium existence. In particular, in the capacitated case $(\bar{D}>k)$, we restrict the function $\theta(x)$ not to be "too concave" to establish an equilibrium, as otherwise the highest possible collusive price may 
not be optimal. In the uncapacitated game, by contrast, we can allow for more general functional forms, and even discontinuities.

Price impacts from a threat of retirement have a similar intuition as those from the penalty function above: deviation incentives are increased as this allows plants to potentially guarantee monopoly profits. But punishments can also increase as the plant can be guaranteed to miss its target and possibly be retired after a deviation which does not take it past its target. However, the overall effect is to decrease prices relative to the stationary game, as a deviating plant also gains in the punishment equilibrium from an increased likelihood of becoming a monopolist in the future.

PROPOSITION 7. In the game with revenue targets and plant retirement,

(a) As distances from the targets increase, prices converge to a strictly lower level than in the game without targets.

(b) When there are no additional financial penalties, prices are lower than those in the stationary game.

(c) In the uncapacitated game, with certain retirement when targets are missed $\left(\theta_{\infty}=\theta_{0}=1\right)$, there is no collusion.

Unlike with the financial penalty, if the deviation incentive dominates, prices with retirement converge to lower price levels, and sometimes to non-collusive pricing when distance from the target increases. This is because deviation incentives created by potential monopoly profits may be such that collusion is not sustained. The effect of retirement is hence qualitatively different from that of penalties, where, with sufficient distance to the target, collusive pricing is maintained. With no capacity constraints and certain retirement of missing plants, collusive prices can in fact never be sustained.

This analysis assumes full "commitment" to mothballing or retiring a plant. With both plants potentially closing, this could leave the market without any peaking units. Commitment in such situations is debatable, and we would likely see some kind of dynamics of mothballing. One way to deal with this issue is to define retirement probabilities jointly, and assign zero probability to the event of both plants retiring. This would not significantly alter the conclusions above. To thoroughly consider this issue, we could allow mothballed plants to re-enter the market. While such a model of industry dynamics is beyond the scope of this paper, this could be accommodated in our model by introducing cyclical demand and formulating penalties as lost revenues during mothballing. Then, the combined impact of targets, penalties and mothballing decisions would yield dynamics for peaking capacity. 
Figure 8 Expected profit for the firm for different revenue targets over time.

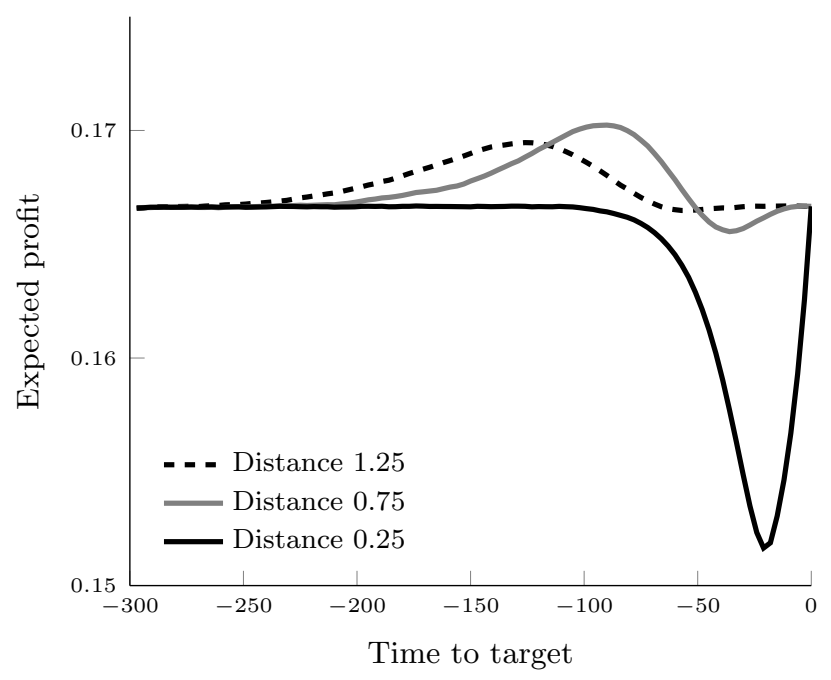

Note. $\bar{P}=1, \bar{D}=1, k=1, \alpha=0.05, \delta=0.96, C=R_{c}$, uniform demand.

\subsection{Discussion of managerial incentives}

The standard argument for firms to impose performance targets is to align shareholder and managerial incentives in the presence of asymmetric information. In our model, no moral hazard due to asymmetric information exists; the performance targets merely act as risk constraints for the plant's operations. In a monopoly, they hence cannot increase the expected profits of risk-neutral owners. But in our duopoly model, we have seen that performance targets can induce higher prices. Consequently, under some conditions, the targets can also be beneficial for (risk-neutral) owners of the firm. This happens in particular when there is sufficient time before the target period and the threat of missing the target serves to decrease the deviation incentives of the plants. However, the potential negative effects for the firm may be large if the target is not set optimally, or if incentives include an implicit threat of closure.

The results are illustrated in Figure 8, where the expected profits for risk-neutral owners are displayed for different revenue targets over time. The owners would prefer to set targets that are relatively far away, both temporally and financially, to put weight on medium-term incentives in pricing. In the peaking plant context, commonly used performance targets for financial years indeed combine these features, allowing enough revenue-earning opportunities for coordination on higher prices. Of course, targets are not usually seen as gaming parameters - they are, at least, financial hurdles to avoid mothballing. Beyond that, however, they could relate to managerial incentives in the context of imperfect competition. 


\section{Portfolio ownership}

In this section we investigate the portfolio effect on the pricing strategies of firms. To do so, we relax the assumption that peakers operate separately from the rest of the market. Building on the model of Section 2, we extend the market setting to cover two technologies that differ in their marginal costs, following Banal-Estañol and Rupérez-Micola (2009). The total capacity $k$ of each generator is divided into baseload (low MC) and peak (high MC) capacity. The portfolio effect is captured by a single parameter $\lambda$, which determines the composition of both firms' plant portfolios.

Let firm F1 own $\lambda k$ baseload (marginal cost $\left.c_{B}=0\right)$ and $(1-\lambda) k$ peak (marginal cost $c_{P}=c$ ) capacity, and firm F2 correspondingly $(1-\lambda) k$ baseload and $\lambda k$ peak capacity, with $\lambda \in[0,1 / 2]$. The portfolio parameter $\lambda$ hence approximates the "diversification" of the market in a simple and intuitive way. This setup ensures that when we vary $\lambda$, (i) the total market capacity is always equally divided into peak and baseload capacity and (ii) each firm's total capacity is kept constant. Thus, $\lambda$ allows us to alter portfolio composition without changing total capacities or the overall market structure. Here F1 is a predominantly "high" marginal cost producer and F2 predominantly "low" cost. Increasing $\lambda$ from zero to one half changes the firms from completely specialized in the two technologies to equally endowed with them. Note that by altering the composition of portfolios of the firms through $\lambda$ we are also changing their effective price-setting power. For example, with $\lambda=0$, the low-MC producer is effectively a monopolist over low demand in one-shot interaction.

We again derive the most-collusive equilibrium sustainable by Nash reversion. Optimal collusion between firms with either asymmetric costs or capacities is in general difficult to characterize (e.g., Lambson 1994, Compte et al. 2002, Miklos-Thal 2011). With heterogeneous generators, there is asymmetry in both costs and capacities, and even the derivation of the one-shot "punishment" equilibrium is difficult because the companies can potentially play different mixed strategies with their low and high cost production. Furthermore, there can in general be many one-shot equilibria with such conflated mixed strategies. We thus limit our analysis to Nash reversion strategies where this behaviour is excluded, since using this simpler equilibrium as punishment provides a sufficient condition to elucidate the general effects of portfolio ownership on collusion.

In the one-shot punishment equilibrium, when demand is low enough, low marginal cost units offer at their marginal cost. With higher demand the equilibrium will be in mixed strategies. The details of the strategies are provided in the Electronic Companion.

Proposition 8. The one-shot punishment equilibrium with portfolio ownership can be characterised as follows.

1. When $D \leq \lambda k$, the firms price their low $M C$ production at 0 .

2. When $\lambda k<D \leq k$, the firms use a mixed strategy to set their low MC production prices below $c$ and set the high $M C$ production prices at $c$. 
3. When $D>k$, the firms use a mixed strategy to set a single price for their entire production at prices below $\bar{P}$.

Next, we will construct the most-collusive equilibrium of the repeated game. Recalling the form of equilibrium from Section 2, the collusive price and thresholds $\left\{\underline{D}^{*}, \bar{D}^{*}\right\}$ will be equal for the firms in this equilibrium. But given the asymmetry between the firms, any $\underline{D}^{*}$ would imply a different $\bar{D}^{* i}$ for the two firms. This suggests that the fully collusive pricing region would be defined as a minimum set for the two firms, and the price similarly as the minimum sustainable price. However, we can show that the predominantly low marginal cost producer (F2) will always be more likely to deviate from the equilibrium, and thus focus on its collusive strategy. From this result, it follows that homogeneous portfolios tend to make implicit collusion easier. Intuitively, this is because with differing portfolios, F2 will have both lower punishments due to higher one-shot profits and higher deviation gains, driven by lower fraction of low MC capacity.

Proposition 9. For the most-collusive symmetric equilibrium described above, the more similar the portfolios, the larger the range of demand values for which full collusion is sustainable.

The result in Proposition 9 is partly driven by two implicit assumptions in the model. First, profits are shared according to capacity, and allowing market share coordination could result in different equilibria. Our approach, however, mirrors the practice of a system operator in an electricity market resulting from its obligation to non-discriminatory treatment of power producers. Second, punishments reverting to the one-shot equilibrium in Proposition 8 yield for some demand ranges profits strictly larger than minmax punishments. ${ }^{2}$ Next, we investigate the sensitivity of our result to this equilibrium selection by repeating the analysis with these punishments.

With minmax punishments, it is no longer clear that firm F2 is always more likely to deviate, and finding the most-collusive equilibrium becomes more difficult. However, a numerical investigation shows that this is still the most plausible outcome. To find an approximate equilibrium, we adopt the following numerical approach.

1. Solve for the optimal $\underline{D}^{*}, \bar{D}^{*}, p_{c}$ for F2.

2. Find the set of demand values $D_{1} \in[0, \bar{D}]$ for which F1 would deviate. In this set, decrease the price $p_{c}$ by an increment and update the set. Repeat until $D_{1}=\emptyset$.

3. Repeat step 2 alternating between F2 and F1 until neither price is revised down at any $D$. Note that since $p_{c}$ is decreased only for some prices, the deviation gain decreases more than punishment in this set and therefore the updating will converge.

Following this approach, we arrive at an equilibrium with the highest possible sustainable price for any $D$. Our numerical experiments showed that the relationship between the heterogeneity of 
Figure 9 The average collusive prices achieved in the two types of equilibria for different $\lambda$.

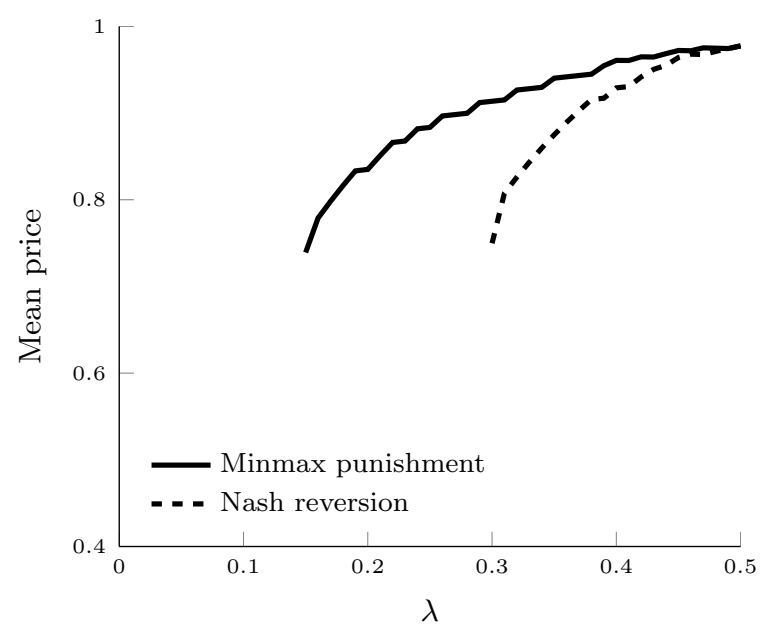

Note. $\delta=0.6, c=0.2, \bar{P}=1, \bar{D}=1, k=0.5$, uniform demand.

producers and the collusive prices continues to hold with minmax punishments. This is demonstrated in Figure 9, where mean collusive prices achieved are plotted against $\lambda$, with a comparison against the Nash reversion case. As expected, collusive outcomes are more likely with minmax punishments, and both prices show increasing trends. Perhaps more interestingly, the collusive outcome may disappear completely when $\lambda$ is small, suggesting a potentially strong impact with changing market structure.

Portfolio owners in electricity markets may thus be more likely (than specialized firms of similar size) to be able to exercise market power by implicitly colluding on prices. In a peaker environment, the baseload and peakers could be for example gas and oil turbines, respectively; this is a fairly accurate description of a present-day balancing power market (with no hydropower), with the difference that gas turbines would often also sell their production forward, potentially competing with other fuel types. To the extent that collusive behaviour is reinforced, we would expect markets to evolve towards peak capacity not only concentrated into relatively few owners, but with these owners holding portfolios of different technologies. This intuition is supported by the data in Figure 10. Out of the thermal plants operating in the UK in 2006-2009, none of the infrequently operated ones were owned by small market participants. (Here, "large" participants include SSE, SP, EON, RWE, IP/GDF and BE). However, the small owner fraction amongst the plants operated almost daily (over 1200 days of producing at least $100 \mathrm{MWh}$ in four years) was about 25\%. This is consistent with the above analysis that peaking facilities can be more effective and sustainable as part of large generating portfolios. 
Figure 10 Plant portfolios: the fraction of thermal plants operating with different frequencies owned by small market participants in the UK electricity market, as well as the total number of thermal plants.

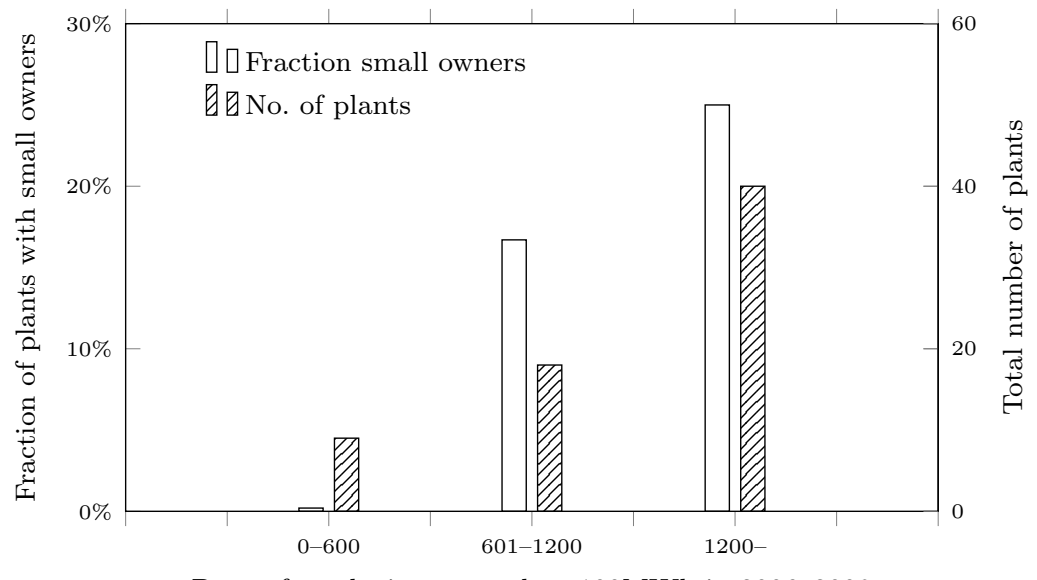

Days of producing more than 100MWh in 2006-2009

\section{Conclusions}

Peak producers' financial viability evaluations need to reconcile potentially significant price setting power with the infrequency of revenue-earning opportunities. In this paper, we have extended a model of repeated price competition to this context. Our model, along with establishing driving factors of peaker price decisions, also provides the underlying process for the formation of price spikes.

Even with infrequent interactions, peak producers may be able to achieve implicitly collusive markups. We have derived the conditions for such equilibria to exist; these depend upon, among others, the frequency of peak events, but also the interplay of available peaking capacity and the distribution of residual peak demand. Depending on this distribution, the existence of a collusive equilibrium may require significant over-capacity in the market, which may be aligned with regulator goals for resource security. Moreover, collusive price spikes may emerge with low residual demand as well as high demand. We presented some indicative evidence for these price developments in the British electricity market.

For financial viability, peak producers are often required to meet performance targets, imposed either from the outside (by stakeholders) or as managerial incentives. These targets induce dynamic pricing patterns even with stationary demand in both collusive and non-collusive pricing. The patterns are non-monotonic and produce distinct predictions: targets may simultaneously increase non-collusive prices and decrease collusive prices. For collusive prices, non-monotonicity results from two intertwined effects: deviation incentives are increased by the opportunity to reduce the shortfall from the target, but further reducing this distance may be difficult in the resulting punishment equilibrium. In particular, temporally fairly spread out, but ambitious targets may encourage 
high prices. Echoing the limited liability effect of Brander and Lewis (1986), optimally-set targets may be beneficial through incentivising managers. By contrast, if targets need to be met to avoid closure or mothballing, the effect on collusive prices is negative: producers may expect either no future profits or monopoly profits, discouraging price coordination. These two discrepancies in the impact of performance targets - between non-collusive and collusive pricing, and between financial penalties and threat of closure - highlight the importance of a detailed understanding of the consequences of performance and risk measures in this setting.

We further found that ownership of both peak and baseload capacity increases the overall amount of collusion in the market and thus profitability. This result suggests that while specialised companies might enjoy scale benefits, portfolio ownership allows them to use market power more effectively. Traditionally, risk mitigation has been considered the main advantage of technological diversification. Here, we conclude that beyond risk considerations, portfolio ownership can significantly benefit the companies by allowing them to (collusively) drive prices higher.

Aside from the operational decisions and asset valuation of peaking plants, our model also offers insights for regulators: in volatile markets, infrequently operating peakers are needed to retain adequate capacity in scarcity and policies to ensure their profitable existence need to balance price restraints with asset returns. Here, we have considered this problem in the conventional "energyonly" market setting. By contrast, complementary markets for long-term options (Oren 2005) or capacity (Cramton and Stoft 2006) can be used to remunerate infrequently operating plants. We provide a counterfactual analysis to these approaches: peakers who choose to participate in such contracts are in essence forgoing market power in the spot market, and the cost of incentivising their participation will reflect this. In particular, this opportunity cost will be greater for larger companies with portfolio diversification.

Emissions targets agreed upon in different countries require that renewable energy production, notably in wind power, should increase significantly in the coming decades. Given the lack of viable large-scale storage options, the intermittency of wind power will increase the volatility of electricity supply, exacerbating the need for flexible, infrequently operating production capacity (e.g., Grave et al. 2012). Understanding (and providing) the financial incentives for peakers' decisions is thus becoming more and more significant. In this paper, we have focused on pricing strategies; however the investment, financing, and mothballing decisions driven by these prices and the resulting potential dynamics of market structure are equally important for the interaction of peak producers. Extending the present work to capacity decisions is hence an important goal for future research.

\section{Endnotes}


1. Offers are made for half-hourly periods an hour in advance, allowing for dynamic strategies within the day.

2. Such a punishment could be achieved through producer-specific punishments with nonstationary time structure ('carrot-and-stick'), see Abreu (1988). In such an equilibrium, the 'stick', or the punishment, may be harsher than the one-shot Nash equilibrium (but temporary).

\section{Acknowledgments}

The authors thank the editor Eddie Anderson, the associate editor and three referees for insightful comments, with Section 3 of the paper in particular greatly benefiting from their ideas. The authors would also like to thank S. Alex Yang for helpful discussions, and various seminar participants for their feedback. Phil Hewitt and Paul Verrill of EnAppSys Ltd provided market data and valuable comments. The first author gratefully acknowledges funding from the Emil Aaltonen Foundation and the Fortum Foundation, and the hospitality of the Systems Analysis Laboratory at Aalto University School of Science, where parts of this research were conducted.

\section{References}

Abreu, Dilip. 1988. On the theory of infinitely repeated games with discounting. Econometrica 56(2) 383-396.

Anderson, E. J., T. D. H. Cau. 2009. Modeling implicit collusion using coevolution. Operations Research $\mathbf{5 7}(2) 439-455$.

Anderson, E. J., T. D. H. Cau. 2011. Implicit collusion and individual market power in electricity markets. European Journal of Operational Research 211(2) 403-414.

Anderson, E. J., A. B. Philpott. 2002. Using supply functions for offering generation into an electricity market. Operations Research 50(3) 477-489.

Banal-Estañol, A., A. Rupérez-Micola. 2009. Composition of electricity generation portfolios, pivotal dynamics and market prices. Management Science 55(11) 1813-1831.

Bernheim, B. D., M. D. Whinston. 1990. Multimarket contact and collusive behavior. The RAND Journal of Economics 21(1) 1-26.

Besbes, O., C. Maglaras. 2012. Dynamic pricing with financial milestones: Feedback-form policies. Management Science 58(9) 1715-1731.

Bolton, P., D. S. Scharfstein. 1990. A theory of predation based on agency problems in financial contracting. The American Economic Review 80(1) 93-106.

Brander, J. A., T. R. Lewis. 1986. Oligopoly and financial structure: the limited liability effect. The American Economic Review 76(5) 956-970.

Bushnell, J. 2003. A mixed complementarity model of hydrothermal electricity competition in the western United States. Operations Research 51(1) 80-93. 
Compte, O., F. Jenny, P. Rey. 2002. Capacity constraints, mergers and collusion. European Economic Review 46(1) $1-29$.

Cramton, P., S. Stoft. 2006. The convergence of market designs for adequate generating capacity. White Paper, California Electricity Oversight Board.

Davidson, C., R. Deneckere. 1990. Excess capacity and collusion. International Economic Review 31(3) $521-541$.

Dechenaux, E., D. Kovenock. 2007. Tacit collusion and capacity withholding in repeated uniform price auctions. The RAND Journal of Economics 38(4) 1044-1069.

Dechenaux, E., D. Kovenock. 2011. Endogenous rationing, price dispersion and collusion in capacity constrained supergames. Economic Theory 47(1) 29-74.

Eckert, V. 2013. Weak demand may force Eon to mothball new power stations. Reuters. Published March 13, 2013. Retrieved from http://www.reuters.com/ 16 August, 2013.

Ernst \& Young. 2013. European power and utility asset impairments. Retrieved from http://www.ey.com/ 27 October, 2014.

Fabra, N. 2006. Collusion with capacity constraints over the business cycle. International Journal of Industrial Organization 24(1) 69-81.

Fabra, N., J. Toro. 2005. Price wars and collusion in the Spanish electricity market. International Journal of Industrial Organization 23(3-4) 155-181.

Fabra, N., N. H. von der Fehr, D. Harbord. 2006. Designing electricity auctions. The RAND Journal of Economics 37(1) 23-46.

Garcia, A., E. Campos-Nañez, J. Reitzes. 2005. Dynamic pricing and learning in electricity markets. Operations Research 53(2) 231-241.

Grave, K., M. Paulus, D. Lindenberger. 2012. A method for estimating security of electricity supply from intermittent sources: scenarios for Germany until 2030. Energy Policy 46 193-202.

Green, R. J., D. M. Newbery. 1992. Competition in the British electricity spot market. Journal of Political Economy 100(5) 929-953.

Guan, X., Y. C. Ho, D. L. Pepyne. 2002. Gaming and price spikes in electric power markets. Power Systems, IEEE Transactions on 16(3) 402-408.

Haltiwanger, J., J. E. Harrington. 1991. The impact of cyclical demand movements on collusive behavior. The RAND Journal of Economics 22(1) 89-106.

Hobbs, B. F. 2001. Linear complementarity models of Nash-Cournot competition in bilateral and POOLCO power markets. Power Systems, IEEE Transactions on 16(2) 194-202.

Hobbs, B. F., J. S. Pang. 2007. Nash-Cournot equilibria in electric power markets with piecewise linear demand functions and joint constraints. Operations Research 55(1) 113-127. 
Jing-Yuan, W., Y. Smeers. 1999. Spatial oligopolistic electricity models with Cournot generators and regulated transmission prices. Operations Research 47(1) 102-112.

Knittel, C. R., J. J. Lepore. 2010. Tacit collusion in the presence of cyclical demand and endogenous capacity levels. International Journal of Industrial Organization 28(2) 131-144.

Lambson, V. E. 1994. Some results on optimal penal codes in asymmetric Bertrand supergames. Journal of Economic Theory 62(2) 444-468.

Levin, Y., J. McGill, M. Nediak. 2008. Risk in revenue management and dynamic pricing. Operations Research 56(2) 326-343.

Macatangay, R.E.A. 2002. Tacit collusion in the frequently repeated multi-unit uniform price auction for wholesale electricity in england and wales. European Journal of Law and Economics 13(3) 257-273.

Maksimovic, V. 1988. Capital structure in repeated oligopolies. The RAND Journal of Economics 19(3) 389-407.

Miklos-Thal, J. 2011. Optimal collusion under cost asymmetry. Economic Theory 46(1) 99-125.

Mount, T.D., Y. Ning, X. Cai. 2006. Predicting price spikes in electricity markets using a regime-switching model with time-varying parameters. Energy Economics 28(1) 62-80.

Ofgem. 2013. Electricity capacity assessment report.

Oren, S.S. 2005. Generation adequacy via call options obligations: safe passage to the promised land. The Electricity Journal 18(9) 28-42.

PR Newswire. 2004. TXU power to mothball gas-fired power plant units; other gas plant operations to be optimized. Published 12 November, 2004. Retrieved from http://www.prnewswire.com/ 16 August, 2013.

Rochet, J-C., S. Villeneuve. 2011. Liquidity risk and corporate demand for hedging and insurance. Journal of Financial Intermediation 20(3) 300-323.

Rotemberg, J. J., G. Saloner. 1986. A supergame-theoretic model of price wars during booms. The American Economic Review 76(3) 390-407.

Staiger, R.W., F.A. Wolak. 1992. Collusive pricing with capacity constraints in the presence of demand uncertainty. The RAND Journal of Economics 23(2) 203-220.

Sweeting, A. 2007. Market power in the England and Wales wholesale electricity market 1995-2000. The Economic Journal 117(520) 654-685.

Talluri, K. T., G. Van Ryzin. 2004. The theory and practice of revenue management. Springer Verlag.

Traber, T., C. Kemfert. 2011. Gone with the wind? - electricity market prices and incentives to invest in thermal power plants under increasing wind energy supply. Energy Economics 33(2) 249-256.

von der Fehr, N. H. M., D. Harbord. 1993. Spot market competition in the UK electricity industry. The Economic Journal 103(418) 531-546. 
Wiesmann, G., P. Clark. 2012. Eon warns on conventional power. Financial Times. Published November 13, 2012. Retrieved from http://www.ft.com/ 16 August, 2013.

Wolfram, C. D. 1998. Strategic bidding in a multiunit auction: an empirical analysis of bids to supply electricity in England and Wales. The RAND Journal of Economics 29(4) 703-725.

\section{Appendix A: Results in Section 2: Model for Peaker Pricing}

\section{A.1. Proof of Lemma 1}

This is a well-known result, and we omit some details. For a more comprehensive treatment, see e.g., Fabra et al. (2006).

If firm $i$ submits an offer $p^{i}$ for its capacity, the quantity it supplies is given by

$$
q^{i}\left(D, p^{i}, p^{j}\right)= \begin{cases}\min \{D, k\} & \text { if } p^{i}<p^{j}, \\ \min \{R D, k\} & \text { if } p^{i}>p^{j}, \\ \frac{D}{2} & \text { if } p^{i}=p^{j}\end{cases}
$$

where $R D=\max \{0, D-k\}$ is the residual demand for firm $i$. In the discriminatory (or pay-as-bid) auction the plants are always paid their own offer price, and therefore the profits are given by

$$
\pi^{i}\left(D, p^{i}, p^{j}\right)=\left(p^{i}-c\right) q^{i}\left(D, p^{i}, p^{j}\right)
$$

If $D \leq k$, prices are set at marginal cost in the equilibrium. Suppose instead that $p^{i}>c=0$ : then firm $j$ will undercut since it can capture the entire market demand. Hence $p^{i}=p^{j}=0$.

If $D>k$, the competitive equilibrium above does not exist, since by eq. (29), any deviating firm would serve a positive amount and thus gain from deviating. Suppose that $p^{i}$ is the highest offer. Now firm $j$ 's profit is strictly increasing up to $p^{i}$. Therefore, in any pure strategy equilibrium, the offers are the same for both firms. If both firms offer $p$, by the profit function (30) they are equally sharing the demand. Since $D>k$, either can gain by just undercutting $p$ to capture $k$. Therefore, no pure strategy equilibrium exists.

We can derive the (unique) mixed strategy equilibrium by setting expected profits equal for all $p<\bar{P}$ :

$$
\begin{aligned}
\pi^{i}(D, p) & =H_{o}^{j}(p)[(p-c)(D-K)-z(C+(p-c)(D-K))] \\
& +\left(1-H_{o}^{j}(p)\right)[(p-c) K-z(C+(p-c) K)]
\end{aligned}
$$

Solving this, we have for $i=1,2$ :

$$
H_{o}^{i}(p)=\frac{(p-c) K-\pi^{j}(D)}{(p-c)(2 K-D)} .
$$

It is obvious that both firms should have the same lower bound of price range. Otherwise, the one with the lower price range should deviate by increasing the lower end of the range to make more profit. Let the lower bound be $\underline{p}$. Setting $H_{o}^{i}(\underline{p})=0$, we have $\pi^{i}(D)=(\underline{p}-c) K$ for $i=1,2$.

On the other hand, we need to have $H_{o}^{i}(\bar{P})=1$ for $i=1,2$. Therefore, $\pi^{i}(D)=(\bar{P}-c)(D-K)$. Substitute this back to $\underline{p}$ and we have the desired lower bound. 


\section{A.2. Proof of Lemma 2}

The generators wish to share monopoly profits. With demand $D$, they would make $D \bar{P} / 2$ each. If $D \leq k$, a deviation yields a gain of $D \bar{P} / 2$ by capturing the whole market demand. If, on the other hand, $D>k$, the firm can only capture the demand up to $k$, gaining $(k-D / 2) \bar{P}$. Thus the deviation gain is increasing in $D$ up to $k$ and decreasing after it.

With $D \leq k$, if the punishment $0 \leq M \leq k \bar{P} / 2$, the deviation gain will be equal to it at some demand, $\underline{D}^{*}$, so that deviation gain exceed the punishment for higher demand realizations. Similarly for $D>k$, as the deviation gain is decreasing in demand, it will exceed the punishment when demand is close to $k$ and will be equal to it at $\bar{D}^{*}$. Since the gains at $\underline{D}^{*}$ and $\bar{D}^{*}$ are equal to $M$, we have $\left(k-\bar{D}^{*} / 2\right) \bar{P}=\underline{D}^{*} \bar{P} / 2$ or equivalently $\underline{D}^{*}+\bar{D}^{*}=2 k$. But since $\bar{D} \leq 2 k$, we also need $\bar{D}^{*} \leq \bar{D}$ or equivalently $\underline{D}^{*} \geq 2 k-\bar{D}$.

Let us derive the optimal punishment. The infinite punishment involves pricing at the one-shot Nash equilibrium strategies for every period after deviation. In any period, a residual demand event arises with probability $\alpha$ and periods are discounted with $\delta$. The punishment is

$$
M=\sum_{t=1}^{\infty}\left[\alpha \delta^{t} \int_{0}^{\bar{D}}\left(\pi_{c}(D)-\pi_{o}(D)\right) d F(D)\right],
$$

where $\pi_{c}$ is the collusive profit and $\pi_{o}$ is the one-shot profit for a single firm. It is clear that $M$ does not depend on the current demand realization. We can write the punishment as

$$
\begin{aligned}
M & =\alpha \frac{\delta}{1-\delta} \int_{0}^{\bar{D}}\left(\pi_{c}(D)-\pi_{o}(D)\right) d F(D) \\
& =\frac{\gamma}{1-\gamma} \int_{0}^{\bar{D}}\left(\pi_{c}(D)-\pi_{o}(D)\right) d F(D)
\end{aligned}
$$

where we have combined the 'discounting' parameters in one: $\gamma=\alpha \delta /(1-\delta+\alpha \delta)$. (Clearly $\gamma \in$ $(0,1)$.)

For $D \leq k$, we have increasing collusive profits until the cutoff demand, then constant after it, that is, $\pi_{c}(D)=\min \left\{D \bar{P} / 2, \underline{D}^{*} \bar{P} / 2\right\}$ and $\pi_{o}(D)=0$. For $D>k$, we have to consider different cases. Throughout, we have the one-shot profits $\pi_{o}(D)=(D-k) \bar{P}$. Over the upper cutoff point, collusive profits are again $D \bar{P} / 2$, under it they would be $\bar{D}^{*} \bar{P} / 2$, with $\bar{D}^{*}=2 k-\underline{D}^{*}$. However, this equilibrium is not always sustainable when the firms are pricing below the price cap $\bar{P}$. Specifically, if the profits made in this equilibrium are lower than those of deviating by raising the price to $\bar{P}$ (which in this symmetric case are equal to the mixed strategy equilibrium profits, see the proof of Proposition 1), the firms would rather choose not to collude, and end up playing the mixed strategy equilibrium. In fact, collusion will never happen when the mixed strategy equilibrium profits are larger than the collusive profits; colluding in these cases would only lower the total punishment 
and consequently not be 'most-collusive'. Here, this happens when $(D-k) \bar{P}>D P(D) / 2$, where the price $P(D)=\underline{D}^{*} \bar{P} /(2 k-D) \leq \bar{P}$ is the highest sustainable collusive price, derived from the deviation gain being constant below $\bar{D}^{*}$. Thus the firms will not collude when

$$
\frac{k \bar{P}}{\bar{P}-P(D) / 2} \leq D \leq \bar{D}^{*}
$$

where we note that the left hand side is always greater than $k$. To see when the mixed strategies are preferred, we can solve the resulting second degree equation for

$$
D=\frac{6 k-\underline{D}^{*} \pm \sqrt{\left(6 k-\underline{D}^{*}\right)^{2}-32 k^{2}}}{4}
$$

where the mixed equilibrium will be played in between the roots $\{\hat{D}, \check{D}\}$ (when they exist). The range exists when the discriminant $\left(6 k-\underline{D}^{*}\right)^{2}-32 k^{2}$ is positive; this happens in the relevant range when $\underline{D}^{*} \leq(6-4 \sqrt{2}) k \approx 0.34 k$. The range $[\hat{D}, \check{D}]$ will always be constrained between $k$ and $\bar{D}^{*}$. For it to exist, it is further required that $\hat{D} \leq \bar{D}$, that is, $3 k / 2-\underline{D}^{*} / 4 \leq \bar{D}$. In the range, we have (expected) one-shot profits $\pi_{c}(D)=\pi_{o}(D)=(D-k) \bar{P}$.

Now we can write out $\pi_{c}(D)$ and $\pi_{o}(D)$ :

$$
\begin{aligned}
& \pi_{c}(D)= \begin{cases}D \bar{P} / 2 & \text { if } \underline{D} \leq D \leq D^{*} \\
\underline{D}^{*} \bar{P} / 2 & \text { if } \underline{D}^{*}<D \leq k \\
D P(D) / 2 & \text { if } k<D \leq \hat{D} \\
(D-k) \bar{P} & \text { if } \hat{D}<D \leq \bar{D} \\
D P(D) / 2 & \text { if } \check{D}<D \leq \bar{D}^{*} \\
D \bar{P} / 2 & \text { if } \bar{D}^{*}<D \leq \bar{D}\end{cases} \\
& \pi_{o}(D)= \begin{cases}0 & \text { if } \underline{D} \leq D \leq k \\
(D-k) \bar{P} & \text { if } k<D \leq \bar{D} .\end{cases}
\end{aligned}
$$

Combining these into the equation (35), we have the optimal punishment. The prices for each case follow from the discussion.

\section{A.3. Proof of Proposition 1}

The most-collusive strategies have been established in Lemma 2. When $\underline{D}^{*}=0$, the deviation gain and the punishment are both zero so this is a solution corresponding to repeating the static equilibrium strategies. A sufficient condition for the existence of another solution is that $h=W-M$ is either (i) negative at $k$ (and possibly increasing at zero) or (ii) decreasing at zero and positive at $k$ (or (iii) negative somewhere in between, which we don't consider here). In case (i), the firms will collude at monopoly prices for all demand realizations, but in case (ii) they will be able to sustain monopoly prices up to the largest solution to the equation. 
For point 1, The firms will always collude when $h(k) \leq 0$, that is

$$
\begin{aligned}
& h(k)=k \bar{P} / 2-\frac{\gamma}{1-\gamma}\left[\int_{0}^{k} D \bar{P} / 2 d F(D)+\int_{k}^{\bar{D}}(k-D / 2) \bar{P} d F(D)\right] \leq 0 \\
& \Longleftrightarrow \gamma \geq \gamma_{H}^{*}=\frac{x}{1+x}
\end{aligned}
$$

where

$$
x=\frac{k / 2}{\int_{0}^{k} D / 2 d F(D)+\int_{k}^{\bar{D}}(k-D / 2) d F(D)},
$$

which we note does not depend on the price cap. The expression can be interpreted as the maximum deviation gain divided by the average deviation gain, which approaches one when the demand is concentrated around $k$. If more extreme demand realisations become more frequent, $x$ increases. Consequently, the threshold for the discount factor $\gamma$ increases as well.

For point 2, we evaluate $h^{\prime}\left(\underline{D}^{*}\right)$.

$$
\begin{aligned}
& h^{\prime}\left(\underline{D}^{*}\right)=\frac{d}{d \underline{D}^{*}}\left[\underline{D}^{*} \bar{P} / 2-\frac{\gamma}{1-\gamma} \int_{0}^{\bar{D}}\left(\pi_{c}(D)-\pi_{o}(D)\right) d F\left(D^{\prime}\right)\right] \\
& =\frac{d}{d \underline{D}^{*}}\left[\underline{D}^{*} \bar{P} / 2-\frac{\gamma}{1-\gamma}\left[\int_{0}^{\underline{D}^{*}} D \bar{P} / 2 d F(D)+\left[F(k)-F\left(\underline{D}^{*}\right)\right] \underline{D}^{*} \bar{P} / 2\right.\right. \\
& +\int_{k}^{\hat{D}}[D P(D) / 2-(D-k) \bar{P}] d F(D) \\
& \left.\left.+\int_{\check{D}}^{\bar{D}^{*}}[D P(D) / 2-(D-k) \bar{P}] d F(D)+\int_{\bar{D}^{*}}^{\bar{D}}[D \bar{P} / 2-(D-k) \bar{P}] d F(D)\right]\right] \\
& =\bar{P} / 2-\frac{\gamma}{1-\gamma}\left[\underline{D}^{*} \bar{P} / 2 f\left(\underline{D}^{*}\right)+\left(F(k)-F\left(\underline{D}^{*}\right)\right) \bar{P} / 2-f\left(\underline{D}^{*}\right) \underline{D}^{*} \bar{P} / 2\right. \\
& +\int_{k}^{\hat{D}} \frac{D \bar{P} / 4}{k-D / 2} d F(D)+\underline{D}^{*} \frac{d \hat{D}}{d \underline{D}^{*}} \frac{\hat{D} \bar{P} / 4}{k-\hat{D} / 2} f(\hat{D})-(\hat{D}-k) \bar{P} f(\hat{D}) \frac{d \hat{D}}{d \underline{D}^{*}} \\
& +\int_{\check{D}}^{2 k-\underline{D}^{*}} \frac{D \bar{P} / 4}{k-D / 2} d F(D)+\underline{D}^{*}\left(\frac{2 k-\underline{D}^{*}}{2 \underline{D}^{*}} f\left(2 k-\underline{D}^{*}\right)-\frac{d \check{D}}{d \underline{D}^{*}} \frac{\check{D} \bar{P} / 4}{k-\check{D} / 2} f(\check{D})\right) \\
& +(\check{D}-k) \bar{P} f(\check{D}) \frac{d \check{D}}{d \underline{D}^{*}}+\bar{P}\left(k-\underline{D}^{*}\right) f\left(2 k-\underline{D}^{*}\right) \\
& \left.-\int_{2 k-\underline{D}^{*}}^{\bar{D}} \frac{D \bar{P} / 2}{k-D / 2} d F(D)+\bar{P} \underline{D}^{*} / 2 f\left(2 k-\underline{D}^{*}\right)\right]
\end{aligned}
$$

where we have simply used the fundamental theorem of calculus to take derivatives over the integral expressions. For $\underline{D}^{*}=0$, most of the terms disappear and this expression simplifies to the following result:

$$
h^{\prime}(0) \leq 0 \Longleftrightarrow F(k) \geq \frac{1-\gamma}{\gamma} \Longleftrightarrow \gamma \geq \frac{1}{1+F(k)} .
$$

That is, if the demand cannot be fully satisfied by one of firms often enough compared to the inverse of the discount factor, the difference is decreasing at zero. 
If the function $h$ is decreasing at zero and positive at $k$, the existence of a fixed point is guaranteed, since clearly the difference is continuous. We note here that this is a sufficient condition, but since the function $h$, if increasing at zero, will not in general be concave, it is not a necessary condition. Therefore, there may be lower values of the discount factor for which a fixed point exists.

The difference between deviation gain $W$ and punishment $M, h=W-M$, must be increasing at the largest fixed point (if it exists in the interior of the interval). As the discount factor $\gamma$ decreases, the punishment decreases and consequently $h$ increases. Hence we must have a lower $\underline{D}^{*}$ to keep $h$ at zero.

\section{Appendix B: Results in Section 3: Revenue Targets}

\section{B.1. Proof of Proposition 2}

With low demand $(D \leq k)$, it is easy to see that marginal cost pricing is the unique equilibrium. With $D>k$, consider the final period before the target, with the targets of the firms are $\Pi_{T}^{1} \geq \Pi_{T}^{2}$ and penalty function $C\left(\Pi_{t}^{i}-x\right)^{+}$. Like in the stationary case, a pure strategy equilibrium cannot exist, so we consider mixed strategies with CDF's $H_{T, n c}^{i}(p)$. Setting the expected utilities equal for all $p$, we have:

$$
u^{i}(T, p)=H_{T, n c}^{j}(p)\left[p(D-k)-C\left(\Pi_{T}^{i}-p(D-k)\right)^{+}\right]+\left(1-H_{T, n c}^{j}(p)\right)\left[p k-C\left(\Pi_{T}^{i}-p k\right)^{+}\right] .
$$

We can solve these to get

$$
\begin{aligned}
& H_{T, n c}^{1}(p)=\frac{p k-C\left(\Pi_{T}^{2}-p k\right)^{+}-u^{2}(T)}{p(2 k-D)+C\left(\Pi_{T}^{2}-p(D-k)\right)^{+}-C\left(\Pi_{T}^{2}-p k\right)^{+}} ; \\
& H_{T, n c}^{2}(p)=\frac{p k-C\left(\Pi_{T}^{1}-p k\right)^{+}-u^{1}(T)}{p(2 k-D)+C\left(\Pi_{T}^{1}-p(D-k)\right)^{+}-C\left(\Pi_{T}^{1}-p k\right)^{+}} .
\end{aligned}
$$

It is obvious that both firms should have the same lower bound of price range. Otherwise, the one with lower price range should deviate increase the lower end of the range to make more profit. Let the lower bound be $\underline{p}$. Setting $H_{T, n c}^{i}(\underline{p})=0$, we have for $i=1,2$ :

$$
u^{i}(T)=\underline{p} k-C\left(\Pi_{T}^{i}-\underline{p} k\right)^{+} .
$$

On the other hand, each CDF needs to be equal to one at the price cap. However, if the targets $\Pi_{T}^{i}$ differ, the limits of $H_{T, n c}^{i}(p)$ as $p$ approaches $v$ are different. Hence only one of the limits can be equal to one, and the other firm's function will have a mass point at $p=\bar{P}$. Otherwise, one of the firms would have $H_{T, n c}^{i}(\bar{P})>1$. Comparing the limits of the distributions, we find that $\lim _{p \uparrow \bar{P}} H_{T, n c}^{1}(p)=1$. Therefore we have

$$
\begin{aligned}
u^{2}\left(T, \Pi_{T}^{2}, D\right) & =\bar{P}(D-k)-C\left(\Pi_{T}^{2}-\bar{P}(D-k)\right)^{+} \\
\underline{p} & =\frac{1}{k}(\bar{P}(D-k)) \\
u^{1}\left(T, \Pi_{T}^{1}, D\right) & =\bar{P}(D-k)-C\left(\Pi_{T}^{1}-\bar{P}(D-k)\right)^{+} .
\end{aligned}
$$


The lower bound for prices is the same as in the case with no revenue targets and the mixed strategy distributions are (weakly) stochastically dominated by their non-constrained counterparts; the game with targets is hence less competitive.

If just firm 1 is below its target, we have as a special case

$$
\begin{aligned}
& H_{T, n c}^{1}(p)=\frac{p k-u^{2}(T)}{p(2 k-D)} \\
& H_{T, n c}^{2}(p)=\frac{p k-C\left(\Pi_{T}^{1}-p k\right)^{+}-u^{1}(T)}{p(2 k-D)+C\left(\Pi_{T}^{1}-p(D-k)\right)^{+}-C\left(\Pi_{T}^{1}-p k\right)^{+}} .
\end{aligned}
$$

Again comparing the limits of the distributions, we have $\lim _{p \uparrow \bar{P}} H_{T, n c}^{1}(p)=1$. The expected utilities are hence

$$
\begin{aligned}
u_{T}^{2}(D) & =\bar{P}(D-k) \\
u_{T}^{1}\left(\Pi_{T}^{1}, D\right) & =\bar{P}(D-k)-C\left(\Pi_{T}^{1}-\bar{P}(D-k)\right)^{+} .
\end{aligned}
$$

The equilibrium profits for the firm over the price cap are thus not affected; the price distribution is changed though.

Next, consider the second to last period before the target. Now, since the expected utilities of the firms in the last period only depend on their own distance to their target, the expected utilities are

$$
\begin{aligned}
u^{1}(T-1, p)= & H_{T-1, n c}^{2}(p)\left(p(D-k)+\delta \mathbb{E}\left[u^{1}\left(T, \Pi_{T-1}^{1}-p(D-k)\right)\right]\right) \\
& +\left(1-H_{T-1, n c}^{2}(p)\right)\left(p k+\delta \mathbb{E}\left[u^{1}\left(T, \Pi_{T-1}^{1}-p k\right)\right]\right) ; \\
u^{2}(T-1, p)= & H_{T-1, n c}^{1}(p)\left(p(D-k)+\delta \mathbb{E}\left[u^{2}\left(T, \Pi_{T-1}^{2}-p(D-k)\right)\right]\right) \\
& +\left(1-H_{T-1, n c}^{1}(p)\right)\left(p k+\delta \mathbb{E}\left[u^{2}\left(T, \Pi_{T-1}^{2}-p k\right)\right]\right) .
\end{aligned}
$$

Again, we can solve these to get

$$
\begin{aligned}
H_{T-1, n c}^{1}(p) & =\frac{p k+\delta \mathbb{E}\left[u^{2}\left(T, \Pi_{T-1}^{2}-p k\right)\right]-u^{2}(T-1)}{p(2 k-D)-\delta \mathbb{E}\left[u^{2}\left(T, \Pi_{T-1}^{2}-p(D-k)\right)\right]+\delta \mathbb{E}\left[u^{2}\left(T, \Pi_{T-1}^{2}-p k\right)\right]} \\
H_{T-1, n c}^{2}(p) & =\frac{p k+\delta \mathbb{E}\left[u^{1}\left(T, \Pi_{T-1}^{1}-p k\right)\right]-u^{1}(T-1)}{p(2 k-D)-\delta \mathbb{E}\left[u^{1}\left(T, \Pi_{T-1}^{1}-p(D-k)\right)\right]+\delta \mathbb{E}\left[u^{1}\left(T, \Pi_{T-1}^{1}-p k\right)\right]}
\end{aligned}
$$

The expected utilities are for firm $i$

$$
u^{i}(T-1)=\underline{p} k+\delta \mathbb{E}\left[u^{i}\left(T, \Pi_{T-1}^{i}-\underline{p} k\right)\right]
$$

Suppose $\lim _{p \uparrow \bar{P}} H_{T-1, n c}^{i}(p)=1$ for either $i$. Then we have

$$
u^{i}(T-1)=\underline{p} k+\delta \mathbb{E}\left[u^{i}\left(T, \Pi_{T-1}^{i}-\underline{p} k\right)\right]=\bar{P}(D-k)+\delta \mathbb{E}\left[u^{i}\left(T, \Pi_{T-1}^{i}-\bar{P}(D-k)\right)\right],
$$


which has a unique solution $\underline{p} k=\bar{P}(D-k)$. Hence the expected utilities of each firm are

$$
u^{i}(T-1)=\bar{P}(D-k)+\delta \mathbb{E}\left[u^{i}\left(T, \Pi_{T-1}^{i}-\bar{P}(D-k)\right)\right]
$$

These are again independent of the other firm's distance from its target.

Replacing the periods $T$ and $T-1$ by $t$ and $t-1$ in the subscripts of the expressions above, we have an induction argument, and the reasoning holds for $\mathrm{T}$ periods. We can thus characterize the dynamic mixed strategy equilibrium as in the Proposition.

\section{B.2. Proof of Proposition 3}

The distribution of prices is as follows:

$$
\begin{aligned}
H_{t, n c}^{i}(p) & =\frac{p k+\delta \mathbb{E}\left[u^{j}\left(t+1, \Pi_{t}^{j}-p k\right)\right]-\bar{P}(D-k)-\delta \mathbb{E}\left[u^{j}\left(t+1, \Pi_{t}^{j}-\bar{P}(D-k)\right)\right]}{p(2 k-D)-\delta \mathbb{E}\left[u^{j}\left(t+1, \Pi_{t}^{j}-p(D-k)\right)\right]+\delta \mathbb{E}\left[u^{j}\left(t+1, \Pi_{t}^{j}-p k\right)\right]} \\
H_{T, n c}^{i}(p) & =\frac{p k-C\left(\Pi_{T}^{j}-p k\right)^{+}-\bar{P}(D-k)+C\left(\Pi_{T}^{j}-\bar{P}(D-k)\right)^{+}}{p(2 k-D)+C\left(\Pi_{T}^{j}-p(D-k)\right)^{+}-C\left(\Pi_{T}^{j}-p k\right)^{+}}
\end{aligned}
$$

1. When, at time $T, \Pi_{T}^{j} \leq p(D-k)$, it is clear that

$$
H_{T, n c}^{i}(p)=\frac{p k-\bar{P}(D-k)}{p(2 k-D)}=H_{o}^{i}(p) .
$$

2. When $p(D-k)<\Pi_{T}^{j} \leq \bar{P}(D-k)$, we have

$$
H_{T, n c}^{i}(p)=\frac{p k-\bar{P}(D-k)}{p(2 k-D)+C\left(\Pi_{T}^{j}-p(D-k)\right)^{+}},
$$

where, compared to the stationary distribution, the denominator is increased, and hence $H_{T, n c}^{i}(p) \leq$ $H_{o}^{i}(p)$.

3. When $\bar{P}(D-k)<\Pi_{T}^{j} \leq p k$,

$$
H_{T, n c}^{i}(p)=\frac{p k-\bar{P}(D-k)+C\left(\Pi_{T}^{j}-\bar{P}(D-k)\right)^{+}}{p(2 k-D)+C\left(\Pi_{T}^{j}-p(D-k)\right)^{+}} .
$$

Suppose the penalty is linear. Then we have $H_{T, n c}^{i}(p)>H_{o}^{i}(p)$ iff

$$
\begin{gathered}
(p k-\bar{P}(D-k)) C\left(\Pi_{T}^{j}-p(D-k)\right)<p(2 k-D) C\left(\Pi_{T}^{j}-\bar{P}(D-k)\right) \\
(\bar{P}-p)(D-k)\left(p k-\Pi_{T}^{j}\right)<0,
\end{gathered}
$$

which requires $p k<\Pi_{T}^{j}$, implying the next scenario. Hence it is never true for linear penalty. Consequently it is not true for convex penalties either, but may be true for concave penalties. 
4. When $p k<\Pi_{T}^{j}$,

$$
H_{T, n c}^{i}(p)=\frac{p k-C\left(\Pi_{T}^{j}-p k\right)^{+}-\bar{P}(D-k)+C\left(\Pi_{T}^{j}-\bar{P}(D-k)\right)^{+}}{p(2 k-D)+C\left(\Pi_{T}^{j}-p(D-k)\right)^{+}-C\left(\Pi_{T}^{j}-p k\right)^{+}} .
$$

We have $H_{T, n c}^{i}(p) \leq H_{o}^{i}(p)$ iff

$$
\begin{array}{r}
(\bar{P}-p)(D-k) C\left(\Pi_{T}^{j}-p k\right)-p(2 k-D) C\left(\Pi_{T}^{j}-\bar{P}(D-k)\right) \\
+(p k-\bar{P}(D-k)) C\left(\Pi_{T}^{j}-p(D-k)\right) \leq 0 .
\end{array}
$$

Plugging in $x=(\bar{P}-p)(D-k), y=p k-\bar{P}(D-k), z_{1}=\Pi_{T}^{j}-p k, z_{2}=\Pi_{T}^{j}-p(D-k), z=$ $\Pi_{T}^{j}-\bar{P}(D-k)$ and noting that

$$
z=\frac{x}{x+y} z_{1}+\frac{y}{x+y} z_{2}
$$

we have the condition

$$
x C\left(z_{1}\right)+y C\left(z_{2}\right)-(x+y) C(z) \geq 0,
$$

which is true iff $C(\cdot)$ is convex.

\section{B.3. Mixed strategies in dynamic collusive game}

Proposition 10. In the dynamic collusive game with revenue targets at time $T$, penalty functions $C_{T}^{i}(x)=C\left(\Pi_{T}^{i}-x\right)^{+}$mixed strategies are played whenever $k<D \leq 2 k$ and at least one firm would deviate to the price cap. The equilibrium in mixed strategies defined recursively as follows:

$$
\begin{aligned}
H_{t, c}^{i}(p) & =\frac{p k+\delta E_{a t}^{j}(p)-\bar{P}(D-k)-\delta E_{a t}^{j}(\bar{P}(D-k) / k)}{p(2 k-D)-\delta E_{p t}^{j}(p)+\delta E_{a t}^{j}(p)} \\
H_{T, c}^{i}(p) & =\frac{p k-C\left(\Pi_{T}^{j}-p k\right)^{+}-\bar{P}(D-k)+C\left(\Pi_{T}^{j}-\bar{P}(D-k)\right)^{+}}{p(2 k-D)+C\left(\Pi_{T}^{j}-p(D-k)\right)^{+}-C\left(\Pi_{T}^{j}-p k\right)^{+}},
\end{aligned}
$$

with $p \in[\bar{P}(D-k) / k, \bar{P}]$, where

$$
\begin{aligned}
& E_{a t}^{j}(p)=\mathbb{E}\left[u^{j}\left(t+1, \Pi_{t}^{j}-p k, \Pi_{t}^{i}-p(D-k)\right) \mid p^{j}>p\right] \\
& E_{b t}^{j}(p)=\mathbb{E}\left[\left[u_{t+1}^{j}\left(\Pi_{t}^{j}-p(D-k), \Pi_{t}^{i}-p k\right) \mid p^{j} \leq p\right] .\right.
\end{aligned}
$$

Proof. The derivation is similar to that of the non-collusive dynamic equilibrium, with the difference that in the "collusive" mixed equilibrium, both firms' future profits affect the structure of strategies and hence profits.

For the first period before the target, consider mixed strategies with CDF's $H_{T, c}^{i}(p)$. Since the targets do not affect the future states of the game, the mixed strategies are exactly the same as above. Therefore we have the expected utilities

$$
\begin{aligned}
u^{2}\left(T, \Pi_{T}^{2}, D\right) & =\bar{P}(D-k)-C\left(\Pi_{T}^{2}-\bar{P}(D-k)\right)^{+} \\
\underline{p} & =\frac{1}{k}(\bar{P}(D-k)) \\
u^{1}\left(T, \Pi_{T}^{1}, D\right) & =\bar{P}(D-k)-C\left(\Pi_{T}^{1}-\bar{P}(D-k)\right)^{+} .
\end{aligned}
$$


For earlier periods, we need to take into account both firms' profit developments. That is, the (different) profit levels resulting from mixed strategies will affect the collusive prices achieved by the firms through the distance to the target.

Let us next consider the second to last period before the target. Now, since the expected utilities of the firms in the last period only depend on both their own distance to their target and the competitor's distance, the expected utilities are

$$
\begin{aligned}
u^{i}(T-1, p)= & \left.H_{T-1, c}^{j}(p)\left(p(D-k)+\delta \mathbb{E}\left[u^{i}\left(T, \Pi_{T-1}^{i}-p(D-k), \Pi_{T-1}^{j}-p^{j} k\right)\right) \mid p^{j} \leq p\right]\right) \\
& +\left(1-H_{T-1, c}^{j}(p)\right)\left(p k+\delta \mathbb{E}\left[u^{i}\left(T, \Pi_{T-1}^{i}-p k, \Pi_{T-1}^{j}-p^{j}(D-k)\right) \mid p^{j}>p\right]\right) .
\end{aligned}
$$

Here, the inner expectations are with respect to the bid of the other player which follows the distribution $H_{T-1}^{j}$. They determine the distance of the competitor from its target. Denoting the expectations $E_{a}^{i}$ and $E_{b}^{i}$ for brevity, we can write $H^{j}$ as:

$$
H_{T-1, c}^{j}(p)=\frac{p k+\delta E_{a}^{i}-u^{i}(T-1)}{p(2 k-D)-\delta E_{b}^{i}+\delta E_{a}^{i}} .
$$

Notice that since the expectations depend on $H_{T-1}^{j}$, this defines the distribution implicitly. The expected utilities are for firm $i$

$$
u^{i}(T-1)=\underline{p} k+\delta E_{a}^{i}(\underline{p}) .
$$

Suppose $\lim _{p \uparrow \bar{P}} H_{T-1, c}^{i}(p)=1$ for either $i$. Then we have

$$
u^{i}(T-1)=\underline{p} k+\delta E_{a}^{i}(\underline{p})=\bar{P}(D-k)+\delta E_{a}^{i}(\bar{P}(D-k) / k),
$$

which has a unique solution $\underline{p} k=\bar{P}(D-k)$. We further note that at $E_{a}^{i}(\underline{p})$, the other firm is confined to bid only $\underline{p}$ and hence there is no need for the expectation. Therefore we can write the expected utilities of each firm as

$$
u^{i}(T-1)=\bar{P}(D-k)+\delta \mathbb{E}\left[u^{i}\left(T, \Pi_{T-1}^{i}-\bar{P}(D-k), u^{j}\left(T, \Pi_{T-1}^{j}-\bar{P}(D-k)^{2} / k\right)\right)\right] .
$$

Replacing the periods $T$ and $T-1$ by $t$ and $t-1$ in the subscripts of the expressions above, we have an induction argument, and the reasoning holds for $\mathrm{T}$ periods. We can thus characterize the dynamic mixed strategy equilibrium as in the Proposition.

\section{B.4. Proof of Proposition 4}

It is clear that there is always at least an equilibrium (the non-collusive one). For the collusive equilibrium, we need to show that it is always optimal for the plants to choose the highest possible collusive price, due to their expected utility being non-decreasing in price. We will show this by induction. 
In the last period before the target $T$, the expected utility $\mathbb{E}\left[u^{i}\left(T, D, \Pi_{T}\right)\right]$ is non-decreasing in price $p$ up to the most-collusive price. This is easy to see if the plants can make the target by colluding: then the game is like the one without targets. The same is true for the case where they cannot make it by colluding or deviating. In the middle range, where they can make it by deviating only, the prices decrease to reduce the deviation gain, and the firms just miss the target: below it expected profits increase in price. Asymmetric cases can be handled similarly.

Suppose that $\mathbb{E}\left[u^{i}\left(\tau+1, D, \Pi_{\tau}\right)\right]=D p / 2+\delta \mathbb{E}_{u}\left[\tau+1, \Pi_{\tau+1}\left(\Pi_{\tau}, D / 2, p\right)\right]$ is non-decreasing in $p$, where $\mathbb{E}_{u}\left[\tau+1, \Pi_{\tau+1}\right]$ is the expected continuation utility at $\tau+1$. That is, suppressing some notation,

$$
\begin{aligned}
& D / 2+\delta \mathbb{E}_{u}^{\prime}\left[\tau+1, \Pi_{\tau+1}\right] \geq 0 \\
& \mathbb{E}_{u}^{\prime}\left[\tau+1, \Pi_{\tau+1}\right] \geq-D / 2 \delta .
\end{aligned}
$$

Now, in period $\tau$,

$$
\begin{aligned}
\mathbb{E}_{u}\left[\tau, \Pi_{\tau+1}\left(\Pi_{\tau}, D_{\tau} / 2, p_{\tau}\right)\right] & =\mathbb{E}\left[\max _{p\left(p_{\tau}\right)}\left\{D_{\tau} p\left(p_{\tau}\right) / 2+\delta \mathbb{E}_{u}\left[\tau+1, \Pi_{\tau+1}\left(\Pi_{\tau}, D_{\tau} / 2, p_{\tau}\right)\right]\right\}\right] \\
& =\mathbb{E}\left[D_{\tau} p^{*}\left(p_{\tau}\right) / 2+\delta \mathbb{E}_{u}\left(\tau+1, \Pi_{\tau+1}\left(\Pi_{\tau}, D_{\tau} / 2, p^{*}\left(p_{\tau}\right)\right)\right)\right]
\end{aligned}
$$

Differentiating, we have

$$
\begin{aligned}
\mathbb{E}_{u}^{\prime}\left[\tau, \Pi_{\tau+1}\left(\Pi_{\tau}, D_{\tau} / 2, p_{\tau}\right)\right] & =\mathbb{E}\left[p^{\prime *}\left(p_{\tau}\right) D_{\tau} / 2+\delta D_{\tau} p^{\prime *}\left(p_{\tau}\right) / 2 \mathbb{E}_{u}^{\prime}\left[\tau+1, \Pi_{\tau+1}\left(\Pi_{\tau}, D_{\tau} / 2, p^{*}\right)\right]\right] \\
& \geq \mathbb{E}\left[p^{\prime *}\left(p_{\tau}\right) D_{\tau} / 2-p^{\prime *}\left(p_{\tau}\right) D_{\tau} / 2\right]=0
\end{aligned}
$$

where the inequality follows from the induction assumption. Hence we have the induction step. With Proposition 10, it follows that the equilibrium strategies in the text are optimal.

Let us denote the stationary price $p_{b}(D)$. To see that there exist $\tau_{l}, \Pi_{l}, D_{l}$ s.t. $p_{c}\left(\tau_{l}, D_{l}, \Pi_{l}\right)<$ $p_{b}(D)$, where $p_{b}$ is the stationary base-case price (without targets), consider the last period $T$ before the target with $D_{l}, \Pi_{l}$ such that $D_{l} \bar{P} \geq \Pi_{l} \geq D_{l} \bar{P} / 2$. There is an increased incentive to deviate compared to the base case, but the punishment is the same. The price is hence never higher than in the stationary case and sometimes strictly lower.

Now suppose that $p_{b}(D)<\bar{P}$ for some $D$. To see that there exists $\tau_{h}, \Pi_{h}, D_{h}$ s.t. $p_{c}\left(\tau_{h}, D_{h}, \Pi_{h}\right) \geq$ $p_{b}(D)$, suppose $\bar{D} \leq k$. Let us fix a (small) distance $x_{0}$ from the target. Consider period $T$ : the collusive profit is $p_{c}\left(T, D, x_{0}\right) D / 2-C\left(x_{0}-p_{c} D / 2\right)^{+}+M$ and the deviation profit is $p_{c}\left(T, D, x_{0}\right) D-$ $C\left(x_{0}-p_{c} D\right)^{+}$. Clearly the price $p_{c}(T, D)$ is never higher than the base case price $p_{b}(D)$. Now consider the period $T-1$. Collusive profit is $p_{c}\left(T-1, D, x_{0}\right) D / 2+\mathbb{E}\left[p_{c}\left(T, D_{T}, x_{0}-p_{c}(T-\right.\right.$ $\left.\left.\left.1, D, x_{0}\right) D / 2\right) D_{T} / 2\right]-\mathbb{E}\left[C\left(x_{0}-p_{c}\left(T-1, D, x_{0}\right) D / 2-\delta p_{c}\left(T, D_{T}, x_{0}-p_{c} D_{T} / 2\right) D_{T} / 2\right)^{+}\right]+\delta M$ and deviation profit is $p_{c}\left(T-1, D, x_{0}\right) D-\delta C\left(x_{0}-p_{c}\left(T-1, D, x_{0}\right) D\right)^{+}$. For $D>0$ and small enough $x_{0}$, 
the penalties over $C$ disappear in both periods, and the price in period $T$ is equal to $p_{b}(D)$. In period $T-1$, there is a positive probability that $D_{T}$ is small enough so that $p_{c}\left(T, D_{T}, x_{0}-p_{c} D_{T} / 2\right)<$ $p_{b}\left(D_{T}\right)$. But by decreasing $x_{0}$, this probability becomes negligible. Hence the price in period $T$ is virtually equal to $p_{b}(D)$, and converges to $p_{b}(D)$ in earlier periods $T-t$ as $t$ increases for any $x \leq x_{0}$. Now fix another slightly higher distance $x_{1}>x_{0}$. The collusive and deviation profits can be written as above. By the argument above, since prices converge to $p_{b}(D)$ for $x \leq x_{0}$, they will also do so for $x \leq x_{1}$. Now fix $x$ and $D$ such that a deviating firm will not reach the target. Collusive profits in period $T-t$ are $p_{c}(T-t, D, x) D / 2+\sum_{t} \delta^{T-t} \mathbb{E}\left[p_{c}\left(T-t, D_{T-t}, x_{T-t}\right) D_{T-t} / 2\right]-\mathbb{E}\left[C\left(x-p_{c}(T-\right.\right.$ $\left.\left.1, D, x) D / 2-\sum_{t} \delta^{T-t} p_{c}\left(T-t, D_{T-t}, x_{T-t}\right) D_{T-t} / 2\right)^{+}\right]+\delta^{T-t} M$. Suppose $p_{b}(D)<\bar{P}$. Increasing $t$, by the convergence shown above, this will converge to $p_{c}(T-t, D, x) D / 2+\sum_{t} \delta^{T-t} q \mathbb{E}\left[p_{b}\left(D_{T-t}\right)\right]+$ $\delta^{T-t} M$, for $q$ (arbitrarily) close to 1 . Deviation profits, which guarantee not reaching the target, are $p_{c}(T-t, D, x) D-\delta^{T-t} C\left(x-p_{c}(T-1, D, x) D\right)^{+}$. Given that $M=\frac{\delta}{1-\delta} \mathbb{E}\left[p_{b}\left(D_{T-t}\right)\right]$, the price will be higher than the stationary price if $\sum_{t} \delta^{T-t} q \mathbb{E}\left[p_{b}\left(D_{T-t}\right)\right]+\delta^{T-t} M+\delta^{T-t} C\left(x-p_{c}(T-1, D, x) D\right)^{+}>$ $M$, that is, $C\left(x-p_{c}(T-1, D, x) D\right)^{+}>\frac{\mathbb{E}\left[p_{b}\left(D_{T-t}\right)\right](q-\delta) z(\delta)}{\delta^{T-t}}$, which will always hold as $q$ converges to 1 . Thus, for low enough $k$, there exists a demand-time state such that the collusive price will exceed the stationary price.

\section{B.5. Proof of Proposition 5}

The proof is by induction. Consider first a single-period game, that is, $T=1$. Suppose that $\Pi_{T}^{i} \neq \Pi_{T}^{j}$. Then, for a given $(\tau, D, \Pi)$, the $p(\tau, D, \Pi)=\min \left\{p^{i}, p^{j}\right\}$, and $\mathbb{E}_{u}[\tau, D, \Pi]$ for the firms is lower than if the targets were equal. If mixed strategies are played, the utility is similarly lower.

Now consider $T=\tau$. Suppose that the above holds for $T=\tau-1$. That is, for games of shorter length, equal targets are optimal. If $\Pi_{\tau}^{i} \neq \Pi_{\tau}^{j}$, for a given $(\tau, D, \Pi), p(\tau, D, \Pi)=\min \left\{p^{i}, p^{j}\right\}$, and the prices are lower than those with equal targets in all future periods as well. Furthermore, punishments are lower for unequal targets. Thus $\mathbb{E}_{u}[\tau, D, \Pi]$ is lower as well.

\section{B.6. Proof of Proposition 6}

Suppose there is a single equal target at time $T$, the expected stationary punishment is $\Delta \pi_{c, s}$, and the expected punishment in the first period is $\Delta \pi_{c, 1}$. Now consider expanding the horizon from the beginning by adding a another target before the current one (the original $T$ becomes $2 T$ ). Suppose that $\Delta \pi_{c, 1}>\Delta \pi_{c, s}$, that is, targets increase expected profits. The incentives of the original target period are unaffected. Note that the non-collusive equilibrium is unaffected. At time $T$ in the first target period, deviation incentives are not changed, but punishment is increased. Collusive prices hence (weakly) increase. The induction argument is simple: if prices increase in period $\tau<T$, punishment increases. Hence prices also increase in $\tau-1$.

This argument applies to adding further targets. If, on the other hand, the target is such that punishment decreases, the effect is the opposite. 


\section{Appendix C: Results for Section 3.2: Plant retirement}

Again we distinguish between the dynamic non-collusive and collusive game. Given probabilities of retirement $\theta\left(\Pi_{t}^{i}\right)$, in the non-collusive game, the mixed strategies in the last period are derived from

$$
\begin{aligned}
u^{i}(T, p)= & H_{T, n c}^{i}(p)\left[p(D-k)-C\left(\Pi_{T}^{i}-p(D-k)\right)^{+}\right. \\
& \left.+\left(1-\theta\left(\Pi_{T}^{i}-p(D-k)\right)\right) \mathbb{E}\left[\theta\left(\Pi_{T}^{j}-p^{j} k\right) R_{m}+\left(1-\theta\left(\Pi_{T}^{j}-p k\right)\right) R_{n c} \mid p^{j} \leq p\right]\right] \\
& +\left(1-H_{T, n c}^{i}(p)\right)\left[p k-C\left(\Pi_{T}^{i}-p k\right)^{+}\right. \\
& \left.+\left(1-\theta\left(\Pi_{T}^{i}-p k\right)\right) \mathbb{E}\left[\theta\left(\Pi_{T}^{j}-p^{j}(D-k)\right) R_{n c}+\left(1-\theta\left(\Pi_{T}^{j}-p^{j}(D-k)\right)\right) R_{m} \mid p^{j}>p\right]\right],
\end{aligned}
$$

where $R_{n c}$ is the expected discountednon-collusive profit if both plants continue and $R_{m}=$ $\sum_{t} \delta^{t} \mathbb{E}[\bar{P} \min \{D, k\}]$ is the expected discounted monopoly profit if the plant continues alone.

First, let us establish conditions for expected profit to be increasing and concave so that highest collusive prices are optimal and the existence of a mixed strategy equilibrium is guaranteed.

Lemma 3. The profit function $x+\mathbb{E}\left[u\left(t, \Pi_{t}^{i}, \Pi_{t}^{j}, x\right)\right]=\mathbb{E}\left[u^{j}\left(t+1, \Pi_{t}^{j}-f^{j}(x), \Pi_{t}^{i}-f^{i}(x)\right)\right]$ is increasing if $\theta^{\prime}\left(\Pi_{t}^{i}-x\right) \leq \bar{\theta}_{1}$.

Proof. We will show the result for $f^{i}(x)=f^{j}(x)=x$ (the collusive profits case; the mixed profits case is similar. We have assumed $\theta$ to be continuous and concave increasing.

The expected utility of the plants is not always increasing in the price they set. In the last period, this can be seen from differentiating the expected profits

$$
\begin{aligned}
\mathbb{E}\left[u^{i}\left(T, D, \Pi_{T}, p\right)\right]= & D p / 2-C\left(\Pi_{T}^{i}-D p / 2\right)^{+} \\
& +\left(1-\theta\left(\Pi_{T}^{i}-D p / 2\right)\right)\left(\theta\left(\Pi_{T}^{j}-D p / 2\right) R_{m}+\left(1-\theta\left(\Pi_{T}^{j}-D p / 2\right)\right) R_{c}\right) .
\end{aligned}
$$

The derivative with respect to $p$ is

$$
\frac{D}{2}+\frac{D}{2} C^{\prime}\left(x^{i}\right)^{+}+\frac{D}{2} \theta^{\prime}\left(x^{i}\right)\left[\theta\left(x^{j}\right) R_{m}+\left(1-\theta\left(x^{j}\right)\right) R_{c}\right]-\frac{D}{2} \theta^{\prime}\left(x^{j}\right)\left[\left(R_{m}-R_{c}\right)\left(1-\theta\left(x^{i}\right)\right)\right]
$$

where we have set $x^{i}=\Pi_{T}^{i}-D p / 2$. Note that the functions are not differentiable at zero, so this does not cover the cases where the firm reaches its target in the last period. However, it is easy to see that the lowest value for the change is achieved when firm $i$ has reached its target but $j$ has not. Then we have simply

$$
\frac{D}{2}\left(1-\theta^{\prime}\left(x^{j}\right)\left[R_{m}-R_{c}\right]\right)
$$

which is negative for high enough $\theta^{\prime}\left(x^{j}\right)$. With concave $\theta(x)$, this can be prevented by having $\lim _{x \downarrow 0} \theta^{\prime}(x) \leq \frac{1}{R_{m}-R_{c}}$. Then we can follow the steps in the proof of Proposition 4 to show that this condition can be used to guarantee the function to be increasing in earlier periods as well. 
The following proposition describes the dynamic non-collusive (punishment) equilibrium.

Proposition 11. In the dynamic non-collusive game with revenue targets at time $T$ and penalty functions $C(x)^{+}$and retirement probability $\theta\left(\Pi_{T}^{i}\right)$, mixed strategies are played whenever $k<D \leq 2 k$. The equilibrium in mixed strategies is defined recursively as follows:

$$
\begin{aligned}
H_{t, n c}^{i}(p) & =\frac{p k+\delta E_{a t}^{i}(p)-\bar{P}(D-k)-\delta E_{b t}^{j}(\bar{P})}{p(2 k-D)-\delta E_{b t}^{j}(p)+\delta E_{a t}^{j}(p)} \\
H_{T, n c}^{i}(p) & =\frac{p k-C\left(\Pi_{T}^{j}-p k\right)^{+}-\bar{P}(D-k)+C\left(\Pi_{T}^{j}-\bar{P}(D-k)\right)^{+}+E_{c T}^{j}(p)-E_{d T}^{j}(\bar{P})}{p(2 k-D)+C\left(\Pi_{T}^{j}-p(D-k)\right)^{+}-C\left(\Pi_{T}^{j}-p k\right)^{+}+E_{c T}^{j}(p)-E_{d T}^{j}(p)},
\end{aligned}
$$

with $p \in[\bar{P}(D-k) / k, \bar{P}]$, where

$$
\begin{aligned}
& E_{a t}^{j}(p)=\mathbb{E}\left[u^{j}\left(t+1, \Pi_{t}^{j}-p k, t+1, \Pi_{t}^{i}-p^{i}(D-k)\right) \mid p^{i}>p\right] \\
& E_{b t}^{j}(p)=\mathbb{E}\left[u^{j}\left(t+1, \Pi_{t}^{j}-p(D-k), t+1, \Pi_{t}^{i}-p^{i} k\right) \mid p^{i} \leq p\right] \\
& E_{c t}^{j}(p)=\left(1-\theta\left(\Pi_{T}^{j}-p(D-k)\right)\right) \mathbb{E}\left[\theta\left(\Pi_{T}^{i}-p^{i} k\right) R_{m}+\left(1-\theta\left(\Pi_{T}^{i}-p k\right)\right) R_{n c} \mid p^{i} \leq p\right] \\
& E_{d t}^{j}(p)=\left(1-\theta\left(\Pi_{T}^{j}-p k\right)\right) \mathbb{E}\left[\theta\left(\Pi_{T}^{i}-p^{i}(D-k)\right) R_{m}+\left(1-\theta\left(\Pi_{T}^{i}-p^{i}(D-k)\right)\right) R_{n c} \mid p^{i}>p\right] .
\end{aligned}
$$

In the collusive game, the mixed strategies in the last period are derived from

$$
\begin{aligned}
u^{i}(T, p)= & H_{T, c}^{i}(p)\left[p(D-k)-C\left(\Pi_{T}^{i}-p(D-k)\right)^{+}\right. \\
& \left.+\left(1-\theta\left(\Pi_{T}^{i}-p(D-k)\right)\right) \mathbb{E}\left[\theta\left(\Pi_{T}^{j}-p^{j} k\right) R_{m}+\left(1-\theta\left(\Pi_{T}^{j}-p k\right)\right) R_{c} \mid p^{j} \leq p\right]\right] \\
& +\left(1-H_{T, c}^{i}(p)\right)\left[p k-C\left(\Pi_{T}^{i}-p k\right)^{+}\right. \\
& \left.+\left(1-\theta\left(\Pi_{T}^{i}-p k\right)\right) \mathbb{E}\left[\theta\left(\Pi_{T}^{j}-p^{j}(D-k)\right) R_{m}+\left(1-\theta\left(\Pi_{T}^{j}-p^{j}(D-k)\right)\right) R_{c} \mid p^{j}>p\right]\right],
\end{aligned}
$$

where $R_{c}$ is the expected collusive profit if poth plants continue and $R_{m}$ is the monopoly profit if the plant continues alone. In previous periods, the expected profit is as follows:

$$
\begin{aligned}
u^{i}(T-1, p)= & H_{T-1, c}^{j}(p)\left(p(D-k)+\delta \mathbb{E}\left[u^{i}\left(T, \Pi_{T-1}^{i}-p(D-k), \Pi_{T-1}^{j}-p^{j} k\right) \mid p^{j} \leq p\right]\right) \\
& +\left(1-H_{T-1, c}^{j}(p)\right)\left(p k+\delta \mathbb{E}\left[u^{i}\left(T, \Pi_{T-1}^{i}-p k, \Pi_{T-1}^{j}-p^{j}(D-k)\right) \mid p^{j}>p\right]\right) .
\end{aligned}
$$

The following proposition describes the collusive mixed strategies.

Proposition 12. In the dynamic collusive game with revenue targets at time $T$ and penalty functions $C(x)^{+}$, and retirement probability $\theta\left(\Pi_{T}^{i}\right)$, mixed strategies are played whenever $k<D \leq 2 k$ and at least one firm would deviate to the price cap. The equilibrium in mixed strategies is defined recursively as follows:

$$
\begin{aligned}
H_{t, c}^{i}(p) & =\frac{p k+\delta E_{a}^{i}(p)-\bar{P}(D-k)-\delta E_{b t}^{j}(\bar{P})}{p(2 k-D)-\delta E_{b t}^{j}(p)+\delta E_{a t}^{j}(p)} \\
H_{T, c}^{i}(p) & =\frac{p k-C\left(\Pi_{T}^{j}-p k\right)^{+}-\bar{P}(D-k)+C\left(\Pi_{T}^{j}-\bar{P}(D-k)\right)^{+}+E_{c T}^{j}(p)-E_{d T}^{j}(\bar{P})}{p(2 k-D)+C\left(\Pi_{T}^{j}-p(D-k)\right)^{+}-C\left(\Pi_{T}^{j}-p k\right)^{+}+E_{c T}^{j}(p)-E_{d T}^{j}(p)}
\end{aligned}
$$


with $p \in[\bar{P}(D-k) / k, \bar{P}]$, where

$$
\begin{aligned}
& E_{a t}^{j}(p)=\mathbb{E}\left[u^{j}\left(t+1, \Pi_{t}^{j}-p k, \Pi_{t}^{i}-p^{i}(D-k)\right) \mid p^{i}>p\right] \\
& E_{b t}^{j}(p)=\mathbb{E}\left[u^{j}\left(t+1, \Pi_{t}^{j}-p(D-k), \Pi_{t}^{i}-p^{i} k\right) \mid p^{i} \leq p\right] \\
& E_{d t}^{j}(p)=\left(1-\theta\left(\Pi_{T}^{j}-p(D-k)\right)\right) \mathbb{E}\left[\theta\left(\Pi_{T}^{i}-p^{i} k\right) R_{m}+\left(1-\theta\left(\Pi_{T}^{i}-p k\right)\right) R_{c} \mid p^{i} \leq p\right] \\
& E_{c t}^{j}(p)=\left(1-\theta\left(\Pi_{T}^{j}-p k\right)\right) \mathbb{E}\left[\theta\left(\Pi_{T}^{i}-p^{i}(D-k)\right) R_{m}+\left(1-\theta\left(\Pi_{T}^{i}-p^{i}(D-k)\right)\right) R_{c} \mid p^{i} \leq p\right] .
\end{aligned}
$$

The proofs of the two above Propositions closely follow the steps of the proof of Proposition 10 and are hence omitted. Note that here the expected profits refer to the expected collusive profits so the equilibrium needs to be solved in conjunction with the full collusive equilibrium.

The final period payoffs are now

$$
\begin{gathered}
\left.R_{c}^{i}\left(\Pi_{T}^{i}\right)=-C\left(\Pi_{T+1}^{i}\right)^{+}+\left(1-\theta\left(\Pi_{T+1}^{i}\right)\right)\right)\left(\theta\left(\Pi_{T+1}^{j}\right) R_{m}+\left(1-\theta\left(\Pi_{T+1}^{j}\right)\right) R_{c}\right) \\
\left.R_{n c}^{i}\left(\Pi_{T}^{i}\right)=-C\left(\Pi_{T+1}^{i}\right)^{+}+\left(1-\theta\left(\Pi_{T+1}^{i}\right)\right)\right)\left(\theta\left(\Pi_{T+1}^{j}\right) R_{m}+\left(1-\theta\left(\Pi_{T+1}^{j}\right)\right) R_{n c}\right)
\end{gathered}
$$

We can again use the mixed strategy result to write the most-collusive strategies recursively through continuation payoffs:

$$
\begin{aligned}
& \mathbb{E}\left[u^{i}\left(\tau, D, \Pi_{\tau}\right)\right]=\left\{\begin{array}{ll}
D p_{c} / 2+\delta \mathbb{E}\left[u^{i}\left(\tau+1, \Pi_{\tau+1}\left(\Pi_{\tau}, D / 2, p_{c}\right)\right)\right] & \text { if } \max _{i} \zeta^{i}=0 \\
(D-k)^{+} \bar{P}+\delta \mathbb{E}\left[u^{i}\left(\tau+1, \Pi_{\tau+1, M}\left(\Pi_{\tau}\right)\right)\right] & \text { o.w. }
\end{array}\right\}\{1, \ldots, T\} \\
& \zeta^{i}\left(\tau, D, \Pi_{\tau}\right)=\mathbb{1}\left(D p_{c} / 2+\delta \mathbb{E}\left[u^{i}\left(\tau+1, \Pi_{\tau+1}\left(\Pi_{\tau}, D / 2, p_{c}\right)\right)\right] \leq\right. \\
& \left.\quad(D-k) \bar{P}+\delta \mathbb{E}\left[u^{i}\left(\tau+1, \Pi_{\tau+1, M}\left(\Pi_{\tau}\right)\right)\right]\right), \forall i \\
& p_{c}=\min _{i} \arg \max _{p\left(\tau, D, \Pi_{\tau}\right)} \mathbb{E}\left[u^{i}\left(\tau, D, \Pi_{\tau}, p\right)\right] \\
& \mathbb{E}\left[u^{i}\left(\tau, D, \Pi_{\tau}, p\right)\right]=D p / 2+\delta \mathbb{E}\left[u^{i}\left(\tau+1, \Pi_{\tau+1}\left(\Pi_{\tau}, D / 2, p\right)\right)\right], \forall i \\
& u^{i}(T+1, D, \Pi)=R_{c}^{i}\left(\Pi_{T+1}^{i}\right) \\
& \mathbb{E}\left[u^{i}\left(\tau, D, \Pi_{\tau}, p\right)\right] \geq \mathbb{E}\left(u_{d e v}^{i}\left(\tau, D, \Pi_{\tau}, p\right)\right) \forall i \\
& \mathbb{E}\left[u_{d e v}^{i}\left(\tau, D, \Pi_{\tau}, p\right)\right]=\min \{k, D\} p+\left[u_{t+1, n c}^{i}\left(\Pi_{\tau+1}\left(\Pi_{\tau}, \min \{k, D\}, p\right)\right)\right] \\
& u_{t, n c}^{i}\left(\Pi_{t}\right)=\delta \mathbb{E}\left[\bar{P}(D-k)^{+}+u_{t+1, n c}^{i}\left(\Pi_{t+1, M}\left(\Pi_{t}\right)\right)\right] \\
& u_{T+1, n c}^{i}\left(\Pi_{T+1}\right)=R_{n c}^{i}\left(\Pi_{T+1}^{i}\right) .
\end{aligned}
$$

Here $\Pi_{\tau}=\left(\Pi_{\tau}^{i}, \Pi_{\tau}^{j}\right)$ denotes the state of the game, updated as $\Pi_{\tau+1}\left(\Pi_{\tau}, x, p\right)=\left(\Pi_{\tau}^{i}-x p, \Pi_{\tau}^{j}-(D-\right.$ $x) p)$. With mixed strategies, the update is $\Pi_{\tau+1, M}\left(\Pi_{\tau}\right)=\left(\Pi_{\tau}^{i}-\bar{P}(D-k)^{+}, \Pi_{\tau}^{j}-\bar{P}(D-k)^{+2} / k\right)$. 


\section{C.1. Proof of Proposition 7}

Letting the distances from the targets $\Pi^{i} \uparrow \infty$, deviations will happen iff

$$
\begin{gathered}
D G+\left(1-\theta_{\infty}\right) \theta_{\infty} R_{m}+\left(1-\theta_{\infty}\right)^{2} R_{n c}>\left(1-\theta_{\infty}\right) \theta_{\infty} R_{m}+\left(1-\theta_{\infty}\right)^{2} R_{c} \\
D G+\left(1-\theta_{\infty}\right)^{2} R_{n c}>\left(1-\theta_{\infty}\right)^{2} R_{c}
\end{gathered}
$$

where $D G=\min \{D / 2, k-D / 2\} p$ denotes the current period deviation gain and $\theta=0$ corresponds to the case of no retirement. As the distances are large, there is no chance of meeting the target and $\theta$ is not affected by $\Pi^{i}$. Clearly, if $\theta_{\infty}>0$, the RHS is decreased more, and collusion becomes more difficult to sustain. We can see that as $\theta_{\infty} \uparrow 1$, collusion cannot be sustained, and pricing will be non-collusive.

To see that prices will be highest with $\theta(x)=0$, we will use induction.

In the last period before the target, deviation becomes more attractive with retirement. This is because firm 1 will deviate iff

$$
D G+\left(1-\theta_{d}^{1}\right) \theta_{d}^{2} R_{m}+\left(1-\theta_{d}^{1}\right)\left(1-\theta_{d}^{2}\right) R_{n c}>\left(1-\theta_{c}^{1}\right) \theta_{c}^{2} R_{m}+\left(1-\theta_{c}^{1}\right)\left(1-\theta_{c}^{2}\right) R_{c}
$$

where in the shorthand, the superscripts denote the firm and subscripts deviation or collusive retirement probabilities. We can write this as the deviation incentives without retirement and an adjustment term:

$$
D G>R_{c}-R_{n c}+\left(1-\theta_{c}^{1}\right) \theta_{c}^{2}\left(R_{m}-R_{c}\right)-\left(1-\theta_{d}^{1}\right) \theta_{d}^{2}\left(R_{m}-R_{n c}\right)-\theta_{c}^{1} R_{c}+\theta_{d}^{1} R_{n c}
$$

where we can easily verify that the adjustment term (last four terms on RHS) is negative since $\theta_{c}^{1} \leq \theta_{d}^{1}, \theta_{c}^{2} \geq \theta_{d}^{2}$ and $R_{m} \geq R_{c} \geq R_{n c}$. Hence prices decrease with retirement.

Now suppose prices are decreased relative to the case of no retirement in all states in each period from $T$ to $T-t$, and consider period $T-t-1$. The corresponding comparison is then (suppressing notation)

$$
D G>\mathbb{E}\left[u_{c}^{i}\right]-\mathbb{E}\left[u_{d}^{i}\right]
$$

By the induction assumption, prices are lower than prices with no retirement in all future periods until the target, and hence (by Lemma 3 ) collusive profits are lower as well. If, in addition, $\mathbb{E}\left[u_{d}^{i}\right]$ is higher than in the no retirement case, the RHS is decreased and we are done. If $\mathbb{E}\left[u_{d}^{i}\right]$ is lower, if we can show that $\mathbb{E}\left[u_{d}^{i}(T-t-1)\right] \geq \mathbb{E}\left[u_{d}^{i}(T-t)\right]$, we have the same result. To see that this holds, we note from above that the expected profits can be written as

$$
\mathbb{E}\left[u_{d}^{i}\left(T-t-1, \Pi^{i}\right)\right]=\delta\left[(1-\alpha) \mathbb{E}\left[u_{d}^{i}\left(T-t, \Pi^{i}\right)\right]+\alpha\left(\bar{P}(D-k)+\mathbb{E}\left[u_{d}^{i}\left(T-t, \Pi^{i}+\bar{P}(D-k)\right)\right]\right] .\right.
$$


In the corresponding stationary game, $\mathbb{E}\left[u_{d}^{i}\left(T-t-1, \Pi^{i}\right)\right]=\mathbb{E}\left[u_{d}^{i}\left(T-t, \Pi^{i}\right)\right]=\mathbb{E}\left[u_{d}^{i}\left(T-t, \Pi^{i}+\right.\right.$ $\bar{P}(D-k))]$. Now, when $\mathbb{E}\left[u_{d}^{i}\left(T-t, \Pi^{i}\right)\right]<\pi_{n c}$, if $\mathbb{E}\left[u_{d}^{i}\left(T-t, \Pi^{i}+\bar{P}(D-k)\right)\right] \geq \mathbb{E}\left[u_{d}^{i}\left(T-t, \Pi^{i}\right)\right]$, this implies that the expected utility must increase.

To see that $\mathbb{E}\left[u_{d}^{i}\left(T-t, \Pi^{i}+\bar{P}(D-k)\right)\right] \geq \mathbb{E}\left[u_{d}^{i}\left(T-t, \Pi^{i}\right)\right]$ when $\mathbb{E}\left[u_{d}^{i}\left(T-t, \Pi^{i}\right)\right]<\pi_{n c}$, we can use a simple induction argument similar to that in Lemma 3 , but adjusted for the expected value function in the punishment equilibrium in the case $\mathbb{E}\left[u_{d}^{i}\left(T-t, \Pi^{i}\right)\right]<\pi_{n c}$. In the last period, when the expected value is lower, it will clearly be higher in the next period. For the induction step, if $\mathbb{E}\left[u_{d}^{i}(T-t, x)\right] \leq \mathbb{E}\left[u_{d}^{i}\left(T-t, x+q_{1}\right)\right]$,

$$
\begin{aligned}
& \mathbb{E}\left[u_{d}^{i}(T-t-1, x)\right]=\delta\left[(1-\alpha) \mathbb{E}\left[u_{d}^{i}(T-t, x)\right]+\alpha \mathbb{E}_{q}\left[q+\mathbb{E}\left[u_{d}^{i}(T-t, x+q)\right]\right]\right] \\
& \leq \delta\left[(1-\alpha) \mathbb{E}\left[u_{d}^{i}\left(T-t, x+q_{1}\right)\right]+\alpha \mathbb{E}_{q}\left[q+\mathbb{E}\left[u_{d}^{i}\left(T-t, x+q+q_{1}\right)\right]\right]\right] \\
& =\mathbb{E}\left[u_{d}^{i}\left(T-t-1, x+q_{1}\right)\right] .
\end{aligned}
$$

We then have the entire argument for prices being lower with retirement, compared with the stationary game.

Consider now the uncapacitated case. To see that there will never be collusion when plants missing targets are retired with certainty, consider the last period to reach the target $t=T$. Suppose that $\Delta \Pi^{\text {min }}=\min \left\{\Delta \Pi^{i}, \Delta \Pi^{j}\right\}>0$, so that both plants are under the target. If $D p / 2<\Delta \Pi^{\text {min }}$ so that neither can reach its target, both will be retired with certainty. Therefore, there is no punishment to create a collusive outcome, and the one-shot equilibrium will always be played. This is true also if just one of the plants cannot reach its target: it has no future and will thus deviate.

Now suppose $\Delta \Pi^{\text {min }}=\min \left\{\Delta \Pi^{i}, \Delta \Pi^{j}\right\}>0$ but that the $D p<\Delta \Pi^{\text {min }}<D p / 2$ so that both can make it by deviating but not by colluding. The plants only have a future if they deviate, so they will. The same is true if just one of the plants can make the target by deviating but not by colluding.

Now suppose $\Delta \Pi^{\text {min }}=\min \left\{\Delta \Pi^{i}, \Delta \Pi^{j}\right\}>0$ and that $D p / 2 \geq \Delta \Pi^{\text {min }}$, so that both can make their targets by colluding. Deviating ensures that the plant will enjoy monopoly profits which are larger than the collusive continuation profits. Therefore the plants will deviate.

Finally, if one of the plants is below the target and the other has reached its target already, the latter one always has an incentive to deviate to enjoy monopoly profits. To conclude, in the last period, there is no collusion. Consequently, there is no punishment for the period $T-1$, and the same reasoning holds. It follows by induction that the plants never collude if at least one of them starts below the target. 


\section{Appendix D: Results in Section 4: Diversification}

\section{D.1. Proof of Proposition 8}

To find single-shot equilibria for the diversified case, let us again divide the demand into regions. We will only consider equilibria where in high demand situations the firms offer their entire capacity with the same price.

Each generating company has capacity $k$ divided into low (0) marginal cost and high $(c)$ marginal cost capacity. let firm F1 have $\lambda k$ baseload capacity and $(1-\lambda) k$ peak capacity, and firm F2 correspondingly $(1-\lambda) k$ baseload capacity and $\lambda k$ peak capacity, with $\lambda \in[0,1 / 2]$.

Part (i): $D \leq \lambda k$. First, when $D \leq \lambda k$, a single low MC plant from either firm may alone serve the whole demand. Therefore the firms will undercut each other to bring the price to 0 .

Part (ii): $\lambda k<D \leq k$. Second, when we have $\lambda k<D \leq k$, the firms will play mixed strategies as follows. High MC plants will offer their marginal cost $c$ and the low MC plants will mix prices lower or equal to $c$. This means that the high MC plants effectively place a price cap of $c$ on the mixed strategies played by the low MC plants. Since both firms have total capacity $k$ and can thus serve the entire demand in this region, a single firm does not have an incentive to deviate to higher prices. Clearly there is no pure strategy equilibrium for the low MC plants due to cyclic offering similarly to the setting in the one-shot game. The equilibrium mixed strategies can be derived using arguments similar to the Appendix of Fabra et al. (2006) (essentially requiring the mixed strategy to make the competitor indifferent over the support) and are as follows.

Denote $G^{i}(p)=\operatorname{Pr}\left(p^{i} \leq p\right)$ the distribution of the mixed strategy of generator $i$. In the equilibrium we have the firms offering with mixed strategies on $[\underline{p}, c]$ with distributions

$$
\begin{aligned}
G^{1}(p) & =\frac{\min \{(1-\lambda) k, D\}(p-\underline{p})}{p(\lambda k+\min \{(1-\lambda) k, D\}-D)} \\
G^{2}(p) & =\frac{\lambda k(p-\underline{p})}{p(\lambda k+\min \{(1-\lambda) k, D\}-D)}
\end{aligned}
$$

forming the unique mixed equilibrium, with $\underline{p}=c(D-\lambda k) / \min \{(1-\lambda) k, D\}$. This corresponds to expected profits of

$$
\begin{gathered}
\pi^{1}=\lambda k \underline{p} \\
\pi^{2}=\min \{(1-\lambda) k, D\} \underline{p}
\end{gathered}
$$

so the predominantly low cost supplier (F2) makes a larger profit on average, as expected. However, notice that it is also held to its minmax profit $c(D-\lambda k)$ in this equilibrium, making this the lowest expected profit equilibrium it could be held to as a result of a punishment in a repeated game. We note that this is not necessarily the only equilibrium in this demand region (an equilibrium could also involve mixing up to the price cap - similarly to the next paragraph) but this equilibrium can be used to hold the other firm to lower profits than a higher-pricing equilibrium and would thus be used to create a higher punishment. 
Part (iii): $D>k$. Finally, when demand exceeds the total capacity of a single firm, the firms will play mixed strategies up to the price cap $\bar{P}$. Again, no pure strategy equilibria can exist due to cyclical undercutting and price increases; also, the derivation is similar to Fabra et al. (2006), so we omit some details. Suppose that the firms offer their entire capacities $k$ at a single price according to $G^{i}(p)$, with common support in the set $[p, \bar{P}]$. Profits for the firms under mixed strategies are

$$
\begin{aligned}
& \pi^{1}(p)=G^{2}(p)\left[(p(D-k)-c \max \{D-k-\lambda k, 0\}]+\left(1-G^{2}(p)\right)[p k-(1-\lambda) c k]\right. \\
& \pi^{2}(p)=G^{1}(p)\left[(p(D-k)-c \max \{D-k-(1-\lambda) k, 0\}]+\left(1-G^{2}(p)\right)[p k-\lambda c k]\right.
\end{aligned}
$$

where in each of the equations, the first term is the case where the firm offers higher than its competitor and vice versa. In the equilibrium, the firms need to be indifferent over their choice of $p$, so that $\pi^{i}(p)=\bar{\pi}^{i} \forall p$. Then we can solve for

$$
\begin{aligned}
G^{1}(p) & =\frac{p k-\lambda c k-\bar{\pi}^{2}}{p(2 k-D)-c(\lambda k-\max \{D-k-(1-\lambda) k, 0\})} \\
G^{2}(p) & =\frac{p k-(1-\lambda) c k-\bar{\pi}^{1}}{p(2 k-D)-c((1-\lambda) k-\max \{D-k-\lambda k, 0\})} .
\end{aligned}
$$

Noting that $G^{i}(\underline{p})=0$, we have

$$
\begin{aligned}
& \bar{\pi}^{1}=\underline{p} k-(1-\lambda) c k \\
& \bar{\pi}^{2}=\underline{p} k-\lambda c k .
\end{aligned}
$$

To find $\underline{p}$, we need to find the values of the distributions at $\bar{P}$ :

$$
\begin{aligned}
& \lim _{p \uparrow \bar{P}} G^{1}(p)=\frac{\bar{P} k-\underline{p} k}{\bar{P}(2 k-D)-c(\lambda k-\max \{D-k-(1-\lambda) k, 0\})} \\
& \lim _{p \uparrow \bar{P}} G^{2}(p)=\frac{\bar{P} k-\underline{p} k}{\bar{P}(2 k-D)-c((1-\lambda) k-\max \{D-k-\lambda k, 0\})} .
\end{aligned}
$$

Note that these are not necessarily equal. Therefore, only the larger of them can be equal to one. To find if the the limit is larger for Firm 1, we need to compare the denominators of the two expressions and check if

$$
\lambda k-\max \{D-k-(1-\lambda) k, 0\}>(1-\lambda) k-\max \{D-k-\lambda k, 0\},
$$

which is never true for $\lambda \leq 1 / 2$. Therefore, we have $G^{2}(\bar{P})=1$ and can solve for

$$
\left.\underline{p}=\frac{1}{k}(\bar{P}(D-k)-c(\max \{D-k(1+\lambda), 0\}-(1-\lambda) k))\right) \geq c,
$$

which can be plugged in the expected profit equations to get

$$
\begin{aligned}
& \bar{\pi}^{1}=\bar{P}(D-k)-c \max \{D-k(1+\lambda), 0\} \\
& \bar{\pi}^{2}=\bar{P}(D-k)-c \max \{D-k(1+\lambda), 0\}+c k(1-2 \lambda) .
\end{aligned}
$$


Again, the predominantly low marginal cost producer makes a larger profit (with equal profits when $\lambda=1 / 2$ as expected). Here, Firm 1 is held to minmax profits. Firm 2 makes larger than minmax profits (here its minmax profits would be equal to $\bar{P}(D-k)-c \max \{D-k(2-\lambda), 0\})$ if $k<D<k(2-\lambda)$. That is, the firm makes larger than minmax profits the more often, the more specialised the firms are.

\section{D.2. Proof of Proposition 9}

Let us derive the deviation gains and punishments for the two asymmetric firms.

Part (i): Deviation gains. Recall that F1 has $\lambda k$ low MC capacity and $(1-\lambda) k$ high MC capacity, while $\mathrm{F} 2$ has $(1-\lambda) k$ low MC capacity and $\lambda k$ high MC capacity, with the fraction $\lambda \leq 1 / 2$. We assume further that in the collusive equilibrium the generators are offering their entire capacities at the same price and are allocated production according to their (equal) capacities.

To find deviation gains, consider current period profits for a firm when colluding or when deviating by undercutting. If $D \leq k$, the firm can capture the entire demand, while for larger $D$ it can only serve its capacity $k$. For F1, these profits are

$$
\begin{aligned}
\pi^{1} & =\left\{\begin{array}{ll}
D \bar{P} / 2-c(D / 2-\lambda k)^{+} & \text {if collude } \\
D \bar{P}-c(D-\lambda k)^{+} & \text {if deviate }
\end{array} \quad(D \leq k)\right. \\
\pi^{1} & =\left\{\begin{array}{ll}
D \bar{P} / 2-c(D / 2-\lambda k)^{+} & \text {if collude } \\
k \bar{P}-c(1-\lambda) k & \text { if deviate }
\end{array} \quad(D>k) .\right.
\end{aligned}
$$

For F2, the corresponding profits are

$$
\begin{aligned}
\pi^{2} & =\left\{\begin{array}{ll}
D \bar{P} / 2-c(D / 2-(1-\lambda) k)^{+} & \text {if collude } \\
D \bar{P}-c(D-(1-\lambda) k)^{+} & \text {if deviate }
\end{array} \quad(D \leq k)\right. \\
\pi^{2} & =\left\{\begin{array}{ll}
D \bar{P} / 2-c(D / 2-(1-\lambda) k)^{+} & \text {if collude } \\
k \bar{P}-c \lambda k & \text { if deviate }
\end{array} \quad(D>k) .\right.
\end{aligned}
$$

The deviation gains can be found as the difference of the deviation and collusive profits. We need to consider the different cases the $(\cdot)^{+}$-functions with respect to the parameters $D$ and $\lambda$.

Consider first the low demand case $D \leq k$. For F1 there are two parameter thresholds for the $(\cdot)^{+}-$ functions: $\lambda=D / 2 k$ and $\lambda=D / k$. For F2 the thresholds are $\lambda=(k-D) / k$ and $\lambda=(k-D / 2) / k$. Therefore we need to find the deviation gains in the different intervals

$$
\begin{aligned}
& F^{1}: \lambda \in\left[0 \ldots \frac{D}{2 k} \ldots \frac{D}{k} \ldots \frac{1}{2}\right] \\
& F^{2}: \lambda \in\left[0 \ldots \frac{k-D}{k} \ldots \frac{k-\frac{D}{2}}{k} \ldots \frac{1}{2}\right] .
\end{aligned}
$$

Comparing the thresholds for the two firms, we have the following:

$$
\frac{k-D}{k}>\frac{D}{2 k} \Longleftrightarrow D<\frac{2 k}{3}
$$




$$
\begin{aligned}
& \frac{k-D}{k}>\frac{D}{k} \Longleftrightarrow D<\frac{k}{2} \\
& \frac{k-\frac{D}{2}}{k}>\frac{D}{2 k} \Longleftrightarrow D<k \\
& \frac{k-\frac{D}{2}}{k}>\frac{D}{k} \Longleftrightarrow D<\frac{2 k}{3} .
\end{aligned}
$$

Using these, we have three demand intervals: $D \in[0 \ldots k / 2 \ldots 2 k / 3 \ldots k]$. For each of these intervals, $\lambda$ also has different intervals:

$$
\begin{aligned}
& 1^{\circ} \quad D \in\left[0, \frac{k}{2}\right]: \quad \lambda \in\left[0 \ldots \frac{D}{2 k} \ldots \frac{D}{k} \ldots \frac{1}{2}\right] \\
& 2^{\circ} \quad D \in\left[\frac{k}{2}, \frac{2 k}{3}\right]: \quad \lambda \in\left[0 \ldots \frac{D}{2 k} \ldots \frac{k-D}{k} \ldots \frac{1}{2}\right] \\
& 3^{\circ} \quad D \in\left[\frac{2 k}{3}, k\right]: \lambda \in[0 \underbrace{\ldots}_{(i)} \frac{k-D}{k} \underbrace{\ldots}_{(i i)} \frac{D}{2 k} \underbrace{\ldots}_{(i i i)} \frac{1}{2}] .
\end{aligned}
$$

This gives us nine intervals in total where to derive the deviation gains for each of the two firms. These are presented in Table 2. It is easy to see that the deviation gain is always (weakly) larger

Table 2 Deviation gains for the low demand case.

\begin{tabular}{cccc}
\hline & & F1 & F2 \\
\hline & (i) & $D(\bar{P}-c) / 2$ & $D \bar{P} / 2$ \\
$1^{\circ}$ & (ii) & $D \bar{P} / 2-c(D-\lambda k)$ & $D \bar{P} / 2$ \\
& (iii) & $D \bar{P} / 2$ & $D \bar{P} / 2$ \\
\hline & (i) & $D(\bar{P}-c) / 2$ & $D \bar{P} / 2$ \\
$2^{\circ}$ & (ii) & $D \bar{P} / 2-c(D-\lambda k)$ & $D \bar{P} / 2$ \\
& (iii) & $D \bar{P} / 2-c(D-\lambda k)$ & $D \bar{P} / 2-c(D-(1-\lambda) k)$ \\
\hline & (i) & $D(\bar{P}-c) / 2$ & $D \bar{P} / 2$ \\
$3^{\circ}$ & (ii) & $D(\bar{P}-c) / 2$ & $D \bar{P} / 2-c(D-(1-\lambda) k)$ \\
& (iii) & $D \bar{P} / 2-c(D-\lambda k)$ & $D \bar{P} / 2-c(D-(1-\lambda) k)$ \\
\hline
\end{tabular}

for F2.

When $D>k$, from the profits above we can find the threshold $D=2(1-\lambda) k$ for F2. For F1 the $(\cdot)^{+}$-functions are always positive. Therefore we have the following deviation gains (Table 3 ). Again, the deviation gain is (weakly) larger for F2. Together, these results suggest that, all else equal, firm 2 would be more likely to deviate from implicit collusion. However, punishments for the firms differ as well.

Table 3 Deviation gains for the high demand case.

\begin{tabular}{ccc}
\hline & F1 & F2 \\
\hline $1^{\circ} D \leq 2(1-\lambda) k:$ & $(\bar{P}-c)(k-D / 2)$ & $(k-D / 2) \bar{P}-c k \lambda$ \\
$2^{\circ} D>2(1-\lambda) k:$ & $(\bar{P}-c)(k-D / 2)$ & $(\bar{P}-c)(k-D / 2)$ \\
\hline
\end{tabular}


The deviation gains can further be used to derive the relationship between collusive thresholds $\underline{D}^{*}$ and $\bar{D}^{*}$. This is done by equating the deviation gains. For F2, we have the following cases:

1. $\underline{D}^{*} \bar{P} / 2=\left(k-\bar{D}^{*} / 2\right) \bar{P}-c k \lambda \Longleftrightarrow \bar{D}^{*}=2 k-\frac{2 c \lambda}{\bar{P}} k-\underline{D}^{*}$

2. $\underline{D}^{*} \bar{P} / 2=\left(k-\bar{D}^{*} / 2\right)(\bar{P}-c) \Longleftrightarrow \bar{D}^{*}=2 k-\underline{D}^{*} \bar{P} /(\bar{P}-c)$

3. $\underline{D}^{*} \bar{P} / 2-c\left(\underline{D}^{*}-(1-\lambda) k\right)=\left(k-\bar{D}^{*} / 2\right) \bar{P}-c k \lambda \Longleftrightarrow \bar{D}^{*}=2 k-\underline{D}^{*}-2\left(k-\underline{D}^{*}\right) c / \bar{P}$

4. $\underline{D}^{*} \bar{P} / 2-c\left(\underline{D}^{*}-(1-\lambda) k\right)=\left(k-\bar{D}^{*} / 2\right)(\bar{P}-c) \Longleftrightarrow \bar{D}^{*}=2 k-\underline{D}^{*} \bar{P} /(\bar{P}-c)+\left(\underline{D}^{*}-(1-\right.$ $\lambda) k) 2 c /(\bar{P}-c)$.

Similarly, for F1, we have

1. $\underline{D}^{*} \bar{P} / 2=\left(k-\bar{D}^{*} / 2\right)(\bar{P}-c) \Longleftrightarrow \bar{D}^{*}=2 k-\frac{\bar{P}}{\bar{P}-c} \underline{D}^{*}$

2. $\underline{D}^{*} / 2(\bar{P}-c)=\left(k-\bar{D}^{*} / 2\right)(\bar{P}-c) \Longleftrightarrow \bar{D}^{*}=2 k-\underline{D}^{*}$

3. $\underline{D}^{*} \bar{P} / 2-c\left(\underline{D}^{*}-\lambda k\right)=\left(k-\bar{D}^{*} / 2\right)(\bar{P}-c) \Longleftrightarrow \bar{D}^{*}=2 k-\frac{\bar{P}}{\bar{P}-c} \underline{D}^{*}+\frac{2 c}{\bar{P}-c}\left(\underline{D}^{*}-\lambda k\right)$.

To find the prices $P(D)$ that the firms would be able to sustain, we again note that the deviation gain must remain constant between the thresholds. Using the deviation gains, we can derive the prices for the two firms:

$$
\begin{aligned}
& \mathrm{F} 1: P(D)=(\bar{P}-c) \frac{\underline{D}^{*} / 2}{k-D / 2}+c \\
& \mathrm{~F} 2: P(D)=(\bar{P}-c) \frac{\underline{D}^{*} / 2}{k-D / 2}+c \quad \text { if } D, \bar{D}^{*} \geq 2 k(1-\lambda) \\
& \mathrm{F} 2: P(D)=\bar{P} \frac{\underline{D}^{*} / 2}{k-D / 2}+c-c \frac{k \lambda}{k-D / 2} \quad \text { if } D<2 k(1-\lambda), \quad \bar{D}^{*} \geq 2 k(1-\lambda) \\
& \mathrm{F} 2: P(D)=\bar{P} \frac{\underline{D}^{*} / 2}{k-D / 2} \quad \text { if } D, \bar{D}^{*}<2 k(1-\lambda) .
\end{aligned}
$$

In the first case of F2, the prices are the same for both firms. In the third case, the price of F2 is lower. In the second case, F2 is lower if $\lambda<1-\frac{\bar{D}^{*}}{2 k}$, but since $\bar{D}^{*} \geq 2 k(1-\lambda)$, this is never true (strictly). Hence F1's price is at least as low in the second case. Therefore it is not clear which of the firms can sustain a higher price at a given demand with the same parameters. We note that the lower of these prices does not in fact depend on $\lambda$ (except through $\underline{D}^{*}$ ). Moreover, as $\lambda$ decreases, holding other things constant, the third case becomes more prevalent, with lower prices more often.

The mixed strategy thresholds $\{\hat{D}, \check{D}\}$ can be derived similarly to Proposition 1 .

Furthermore, there may be another range $\{\dot{D}, \ddot{D}\}$ in which mixed strategies are preferred when $\frac{k}{2}<D \leq k$. This happens when the mixed strategy profits in this region exceed those with the collusive prices. The mixed strategy profits are

$$
\begin{aligned}
\pi^{1} & =\frac{c \lambda k(D-\lambda k)}{\min \{(1-\lambda) k, D\}} \\
\pi^{2} & =c(D-\lambda k)
\end{aligned}
$$


These never exceed the monopoly profits $D \bar{P} / 2$ but may exceed $\underline{D}^{*} \bar{P} / 2$ when the threshold is low, for example for F2:

$$
c(D-\lambda k)>\underline{D}^{*} \bar{P} / 2 \Longleftrightarrow D>\underline{D}^{*} \frac{\bar{P}}{2 c}+\lambda k .
$$

Note that all else equal, this happens less often with higher $\lambda$.

Part (ii): Punishments. The punishments for the two firms depend on the difference of the collusive profits and the one-shot equilibrium profits that the firms would gain after deviation. Recall that the punishment can be written as

$$
M=\frac{\gamma}{1-\gamma} \int_{0}^{\bar{D}}\left(\pi_{c}(D)-\pi_{o}(D)\right) d F(D)
$$

We have already specified $\pi_{o}$ in the one-shot equilibrium results of Proposition 8. It remains to write down the collusive profits $\pi_{c}$ and combine these two.

Consider first the collusive profits for small demand realisations $D \leq k$. The collusive profits for F1 depend on how much of its production share it has to cover with high MC capacity. They can be written as

$$
\begin{aligned}
\pi_{c}^{1} & =\left\{\begin{array}{ll}
D \bar{P} / 2 & \text { if } D \leq \underline{D}^{*} \\
\underline{D}^{*} \bar{P} / 2-c(D / 2-\lambda k)^{+} & \text {if } D>\underline{D}^{*}
\end{array} \quad\left(\underline{D}^{*} \leq 2 \lambda k\right)\right. \\
\pi_{c}^{1} & =\left\{\begin{array}{ll}
D \bar{P} / 2-c(D / 2-\lambda k)^{+} & \text {if } D \leq \underline{D}^{*} \\
\underline{D}^{*} \bar{P} / 2-c(D / 2-\lambda k) & \text { if } D>\underline{D}^{*}
\end{array} \quad\left(\underline{D}^{*}>2 \lambda k\right) .\right.
\end{aligned}
$$

The corresponding profits for F2 are simple since the firm has enough low MC capacity to always cover half of the demand in this region.

$$
\pi_{c}^{2}=\left\{\begin{array}{ll}
D \bar{P} / 2 & \text { if } D \leq \underline{D}^{*} \\
\underline{D}^{*} \bar{P} / 2 & \text { if } D>\underline{D}^{*}
\end{array} \quad\left(\underline{D}^{*} \leq k\right)\right.
$$

When $D>k$, we have

$$
\begin{aligned}
\pi_{c}^{1} & = \begin{cases}D P(D) / 2-c(D / 2-\lambda k) & \text { if } D \leq \bar{D}^{*} \\
D \bar{P} / 2-c(D / 2-\lambda k) & \text { if } D>\bar{D}^{*}\end{cases} \\
\pi_{c}^{2} & = \begin{cases}D P(D) / 2-c(D / 2-(1-\lambda) k)^{+} & \text {if } D \leq \bar{D}^{*} \\
D \bar{P} / 2-c(D / 2-(1-\lambda) k)^{+} & \text {if } D>\bar{D}^{*}\end{cases}
\end{aligned}
$$

where the maximum sustainable price $P(D)$ can be derived in each case similarly to the symmetric case of Proposition 1.

Taking the difference of the collusive and one-shot profits, we can derive the punishments for the firms. Again, whenever the difference would be negative, the firms would prefer to play mixed strategies of the one-shot equilibrium. 
For F1, the difference is

$$
\pi_{c}^{1}-\pi_{o}^{1}= \begin{cases}D \bar{P} / 2-c(D / 2-\lambda k)^{+}-c(D-\lambda k)^{+} \lambda k / \min \{(1-\lambda) k, D\} & \text { if } D \leq D^{*} \\ \underline{D}^{*} \bar{P} / 2-c(D / 2-\lambda k)^{+}-c(D-\lambda k)^{+} \lambda k / \min \{(1-\lambda) k, D\} & \text { if } \underline{D}^{*}<D \leq k \\ D P(D) / 2-c(D / 2-\lambda k)-\bar{P}(D-k)+c(D-k(1+\lambda))^{+} & \text {if } k<D \leq \bar{D}^{*} \\ D \bar{P} / 2-c(D / 2-\lambda k)-\bar{P}(D-k)+c(D-k(1+\lambda))^{+} & \text {if } D \geq \bar{D}^{*}\end{cases}
$$

For F2, we have the difference

$$
\pi_{c}^{2}-\pi_{o}^{2}= \begin{cases}D \bar{P} / 2-c(D-\lambda k)^{+} \quad \text { if } D \leq \underline{D}^{*} \\ \underline{D}^{*} \bar{P} / 2-c(D-\lambda k)^{+} \quad \text { if } \underline{D}^{*}<D \leq k \\ D P(D) / 2-c(D / 2-(1-\lambda) k)^{+}-\bar{P}(D-k)+c(D-k(1+\lambda))^{+}-(1-2 \lambda) c k & \text { if } k<D \leq \bar{D}^{*} \\ D \bar{P} / 2-c(D / 2-(1-\lambda) k)^{+}-\bar{P}(D-k)+c(D-k(1+\lambda))^{+}-(1-2 \lambda) c k & \text { if } D \geq \bar{D}^{*} .\end{cases}
$$

Based on these, we can compare which of the firms has the larger punishment, all else equal. This is straightforward so we omit most of the details. As an example, let us check whether $\pi_{c}^{1}-\pi_{o}^{1} \geq \pi_{c}^{2}-\pi_{o}^{2}$ for the case where $D \geq \bar{D}^{*}$ (the case $k<D \leq \bar{D}^{*}$ is similar):

$$
\begin{aligned}
& D \bar{P} / 2-c(D / 2-\lambda k)-\bar{P}(D-k)+c(D-k(1+\lambda))^{+} \\
& \geq D \bar{P} / 2-c(D / 2-(1-\lambda) k)^{+}-\bar{P}(D-k)+c(D-k(1+\lambda))^{+}-(1-2 \lambda) c k \\
& \Longleftrightarrow-c(D / 2-\lambda k) \geq-c(D / 2-(1-\lambda) k)^{+}-(1-2 \lambda) c k
\end{aligned}
$$

Suppose that the $(\cdot)^{+}$-function is zero, that is, $D \leq 2 k(1-\lambda)$. Note that we also have $D \geq k$. Then the inequality holds if

$$
\begin{gathered}
D / 2-\lambda k \leq(1-2 \lambda) k \\
\Longleftrightarrow D \leq 2 k(1-\lambda),
\end{gathered}
$$

that is, always when the $(\cdot)^{+}$-function is zero. On the other hand, when this function is positive, the two differences are equal.

Consider next the case where $\underline{D}^{*}<D \leq k$ and $D>\lambda k$ and suppose further that $D>2 \lambda k$ :

$$
\begin{aligned}
& \underline{D}^{*} \bar{P} / 2-c(D / 2-\lambda k)^{+}-c(D-\lambda k) \frac{\lambda}{1-\lambda} \geq \underline{D}^{*} \bar{P} / 2-c(D-\lambda k) \\
& \Longleftrightarrow-c(D / 2-\lambda k)-c(D-\lambda k) \frac{\lambda}{1-\lambda} \geq-c(D-\lambda k) \\
& \Longleftrightarrow D(3 \lambda-1) \leq 2 \lambda^{2} k \\
& \Longleftrightarrow D \leq \frac{2 \lambda^{2}}{3 \lambda-1} k \quad\left(\lambda>\frac{1}{3}\right) .
\end{aligned}
$$

Here, the inequality is reversed if $\lambda<\frac{1}{3}$ and clearly holds then. The multiplier for $k$ on the RHS is always larger than one when $\lambda \in\left(\frac{1}{3}, 1 / 2\right]$ so the inequality always holds. 
Going through all the possible cases in a similar way, in fact we find that

$$
\pi_{c}^{1}-\pi_{o}^{1} \geq \pi_{c}^{2}-\pi_{o}^{2} \quad \forall D
$$

that is, the difference between the collusive and one-shot profits is at least as large (and sometimes strictly larger) for F1 for any demand realisation (given the same parameters and thresholds). Since the demand realisations are the same for both firms, we can thus conclude that, all else equal, the punishment for F1 from deviating is larger than that for F2. This suggests that F2 would be more likely to deviate than F1. We note that lower punishment also means using mixed strategies on a wider range of demand realisations.

Part (iii): Combining the results. We know from above that, all parameters and thresholds equal, the predominantly low marginal cost generator F2 is more likely to deviate. Further, we know that the prices and thresholds are in fact equal for the two firms in the collusive equilibrium. Therefore, we can focus on the incentives of F2. Increasing the diversification parameter $\lambda$ affects collusion in several ways. Here we will show that if a collusive equilibrium exists for $\lambda_{1}$, an equilibrium with the same or more collusive thresholds and prices will exist for a $\lambda_{2}>\lambda_{1}$.

Let us assume that there exists an equilibrium with $\lambda$ with collusive thresholds and prices shared by the two generators. First, we note that the punishment for F2 is non-decreasing in $\lambda$. This can be seen from equation (173). This also means that the area where mixed strategies are played decreases when $\lambda$ increases. Similarly, we can see from Tables 2 and 3 that the deviation gain for F2 is non-increasing in $\lambda$. Moreover, it can be seen from the derivation of collusive thresholds above that the area of demand for which the firms perfectly collude is non-decreasing in the diversification parameter $\lambda$. Finally, above we noted that the maximum price that can be sustained in the equilibrium, $p_{c}$, is non-decreasing in $\lambda$.

Combining these effects, if a collusive equilibrium exists for $\lambda_{1}$, an equilibrium with the same or more collusive thresholds and prices will exist for a $\lambda_{2}>\lambda_{1}$. That is, with higher $\lambda_{2}$, both the firms can still support collusion with the original equilibrium parameters and thresholds. 